\title{
Filamentary Velocity Scaling Validation in the TCV Tokamak
}

\author{
C.K. Tsui ${ }^{1,2}$, J.A. Boedo ${ }^{1}$, J.R. Myra ${ }^{3}$, B. Duval ${ }^{2}$, B. Labit ${ }^{2}$, C. Theiler ${ }^{2}$, N. Vianello ${ }^{4}$, W.A.J. Vijvers ${ }^{5}$, H. \\ Reimerdes $^{2}$, S. Coda ${ }^{2}$, O. Février ${ }^{2}$, J. Harrison ${ }^{6}$, J. Horacek ${ }^{7}$, B. Lipschultz ${ }^{8}$, R. Maurizio ${ }^{2}$, F. Nespoli ${ }^{2}$, U. \\ Sheikh ${ }^{2}$, K. Verhaegh ${ }^{8,2}$, N. Walkden ${ }^{6}$, the TCV Team ${ }^{9}$, and the EUROfusion MST1 Team ${ }^{10}$ \\ 1 University of California San Diego (UCSD), Center for Energy Research (CER), La Jolla, CA 92093- \\ 0417, USA \\ 2 Ecole Polytechnique Fédérale de Lausanne (EPFL), Swiss Plasma Center (SPC), CH-1015 Lausanne, \\ Switzerland \\ 3 Lodestar Research Corporation, Boulder, 2400 Central Avenue, Bolder, Colorado 80301, USA \\ 4 Consorzio RFX (CNR, ENEA, INFN, Universitá di Padova, Acciaierie Venete SpA), Corso Stati Uniti 4, \\ 35127 Padova, Italy \\ 5 FOM Institute DIFFER ‘Dutch Institute for Fundamental Energy Research', De Zaale 20, 5612 AJ \\ Eindhoven, Netherlands \\ 6 CCFE, Culham Science Centre, Abingdon, Oxon, OX14 3DB, United Kingdom \\ 7 IPP.CR, Institute of Plasma Physics AS CR, Za Slovankou 3, 18221 Praha 8, Czech Republic \\ 8 York Plasma Institute, University of York, York YO10 5DQ, UK \\ 9 See author list of S. Coda et al 2017 Nucl. Fusion 57102011 \\ 10 See author list of H. Meyer et al 2017 Nucl. Fusion 57102014
}

\section{Corresponding Author: C7Tsui@ucsd.edu}

\begin{abstract}
A large database of reciprocating probe data from the edge plasma of TCV (Tokamak à Configuration Variable) is used to test the radial velocity scalings of filaments from analytical theory [J. R. Myra, D. A. Russell, and D. A. D'Ippolito, Phys. Plasmas 13, 112502 (2006)]. The measured velocities are mainly scattered between zero and a maximum velocity which varies as a function of size and collisionality in agreement with the analytical scalings. The scatter is consistent with mechanisms that tend to slow the velocity of individual filaments. While the radial velocities were mainly clustered between 0.5 and $2 \mathrm{~km} / \mathrm{s}$, a minority reached outward velocities as high as $5 \mathrm{~km} / \mathrm{s}$ or inward velocities as high as $-4 \mathrm{~km} / \mathrm{s}$. Inward moving filaments are only observed in regions of high poloidal velocity shear in discharges with BxVB away from the $X$-point, a new finding. The filaments have diameters clustered between 3 and $11 \mathrm{~mm}$, and normalized sizes $\hat{a}$ clustered between 0.3 and 1.1, such that most filaments populate the resistive-ballooning regime, therefore, most filaments in TCV have radial velocities with little or no dependence on collisionality. Improvements in crosscorrelation techniques and conditional averaging techniques are discussed which reduce the sizes determined for the largest filaments, including those larger than the scrape-off layer (SOL).
\end{abstract}

\section{Introduction}

The radial transport in the tokamak edge and ScrapeOff Layer (SOL) is turbulence-dominated[1-7]. This is clearly demonstrated by the L-mode to H-mode transition where the quenching of edge turbulence is correlated $[8,9]$ to a decrease in radial transport and increased confinement[10], forming the pedestal and resulting in steep profiles in the SOL[11,12], which lead to very high heat and particle fluxes at the divertor plate that will exceed the engineering limits in future devices. On the other hand, an increase in the turbulent transport is associated with a strong broadening of the far-SOL resulting in a wide and flat density profile called the "density shoulder" which can substantially increase the particle flux arriving at the first wall[13-18]. Turbulence can also affect the momentum[19,20], and energy[16] fluxes throughout the SOL, so a detailed understanding of turbulent transport is necessary in order to develop predictive SOL models.

The turbulence in the edge and SOL consists of broadband plasma density fluctuations with correlated fluctuations in potential and temperature [5,6,21-23]. The largest turbulent events were named 'filaments' from their elongated field-aligned appearance when first observed using fast cameras[24] as well as 'blobs' from their $2 \mathrm{~d}$ appearance as measured using probe arrays[22,25] and Gas Puff Imaging (GPI)[26]. Curvature drifts and VB drifts drive electrostatic polarization within the 
filament causing them to advect radially outward by the ExB drift, [27,28]. The radial motion of these filaments has been shown to strongly enhance crossfield transport with the largest $\sim 1 \%$ of turbulent fluctuations accounting for $\sim 50 \%$ of the cross-field radial ExB convection in the scrape-off layer[29-31].

In order to understand filamentary transport, a model was developed by the progressive work of many theoreticians[27,32-41] and was used to create a radial velocity scaling, which was first described in ref [32]. The model and associated velocity scaling represent a fairly comprehensive understanding of the filament dynamics, but the scaling is based on reduced models and ignores many known processes. For example, filament-neutral interaction can act to increase [42,43] or decrease[44] filament velocity depending on the contribution from charge-exchange neutrals. Filaments can interact with each other when they are in close proximity, causing the electric fields to merge or cancel each other out [45]. The background plasma density has a slowing effect[46,47]. Hot filaments can form a net monopole potential which induces filament rotation, mixing the filament dipoles and slowing them down $[48,49]$.

Kinetic effects can modify the parallel electron response and introduce drift wave dynamics[50]. Parallel variation along the filaments and magnetic shear introduces 3D physics[51-54]. High ion temperature increases the filament drive and modifies the vorticity[55-57]. Regions of diamagnetic and paramagnetic plasma are known to attract and repel filaments[58]. The ellipticity of the filament can act to increase or decrease the radial velocity depending on the tilt of the ellipse[59]. And finally, recent works have also included the role of ion kinetic physics $[60,61]$ and strong density gradients[62,63]. It is hoped that future models that use tested velocity scalings with some statistical spread due to these processes could be successful on increasing model predictability. Such stochastic models are currently under development [64].

The goal of this paper, therefore, is to test the velocity scalings from [32] and to provide information about the statistical spread caused by the other processes. A comprehensive database of thousands of samples is used in order to provide a statistically relevant comparison between experimental data and theory.

We extend previous studies of turbulence in the Tokamak à Configuration Variable (TCV)[17,65-71] by characterizing the size and velocities of the filaments over a range of collisionality. Our paper complements other comprehensive filament velocity scaling studies performed in basic plasma devices[47,72]. More recently, an experimental study of filament motion in NSTX based on GPI data was carried out[73], and a few comparisons with theoretical predictions were made. In contrast to the probe measurement presented here, the GPI analysis provides spatial resolution in two dimensions, but the density and temperature of individual filaments were not available.

\section{$1.1 \quad$ Background}

Filaments have been modeled as equivalent electrical circuits with a voltage source located at the outer midplane (OMP) and a resistive return path located downstream $[37,38]$. The velocity scaling was thus developed by balancing the polarizing voltage source against several distinct return current paths. The normalized radial velocity of the filament is defined as in [39],

$$
\hat{v}=\frac{v_{r}}{\left(\frac{2 L_{\|} \rho_{s}^{2}}{R^{3}}\right)^{1 / 5} c_{s}}
$$

which depends on two main normalized parameters; the normalized filament radius [40], which is described by two related variables,

$$
\hat{a}=\frac{a_{b} R^{1 / 5}}{L_{\|}^{2 / 5} \rho_{s}^{4 / 5}} \text { or } \Theta=\hat{a}^{5 / 2}
$$

and the dimensionless collisionality[41], which one would ideally calculate as an integral along the field line between the OMP and the outer target.

$$
\Lambda=\int_{\text {outer target }}^{O M P} \frac{v_{e i}}{\Omega_{e} \rho_{s}} d s_{\|}
$$

Where $\mathrm{v}_{\mathrm{r}}$ is the radial filament velocity, $\mathrm{s}_{\|}$is the parallel distance from the target, $\mathrm{L}_{\|}$is the parallel connection length from the OMP to the outer target, $\rho_{\mathrm{s}}$ is the ion gyroradius, $\mathrm{R}$ is the major radius, $\mathrm{c}_{\mathrm{s}}$ is the sound speed, $a_{b}$ is the filament radius, $v_{e i}$ is electronion collision frequency, and $\Omega_{\mathrm{e}}$ is the electron gyrofrequency. In this paper, we will assume the ion temperature $\mathrm{T}_{\mathrm{i}}=\mathrm{T}_{\mathrm{e}} . \Lambda$ is used to determine the parallel extent of the filament towards the outer target, i.e. whether the filament's electrical dipole terminates before reaching the $\mathrm{x}$-point, if it reaches into the divertor, or if it extends all the way to the target. Unfortunately, the plasma parameters are not known as a function of $s_{\|}$, so we will consider two estimates for $\Lambda$ following the definitions in [74]. $\Lambda_{\text {mid }}$ will be 
calculated using the full connection length and the plasma parameters measured at the OMP by the reciprocating probe, and $\Lambda_{\text {div }}$ will be calculated using the conditions measured in the divertor by the target Langmuir probes.

$$
\Lambda_{\text {mid }}=\left.L_{\|} \frac{v_{e i}}{\Omega_{e} \rho_{s}}\right|_{O M P} \quad \Lambda_{d i v}=\left.L_{\|}^{x-p t} \frac{v_{e i}}{\Omega_{e} \rho_{s}}\right|_{t a r g}
$$

where $\mathrm{L}_{\|}^{\mathrm{x}-\mathrm{pt}}$ is the parallel distance from the X-point to the outer target. $\Lambda_{\text {div }}$ is meant to represent the collisionality from the high-density region just in front of the target, which can dominate the entire system.

The magnetic field line fanning parameter $\epsilon_{\mathrm{x}}$ represents the effects of elliptical magnetic distortion and from magnetic shear [33]. We will take an approximate value of $\epsilon_{\mathrm{x}} \sim 0.5$ for limited plasmas and $\epsilon_{\mathrm{X}} \sim 0.3$ for diverted plasmas based on the maximum poloidal flux expansion $f_{x \max }$ typically found for those shapes when averaged across the SOL. Note that $\epsilon_{\mathrm{x}}$ is strictly less than one.

$$
\varepsilon_{x} \sim \frac{1}{f_{x \max }} \text { for } f_{x \max }>1
$$

The velocity scaling is defined for four regimes in the $\Lambda$ - $\hat{a}$ space shown in Figure 1 and each regime has a characteristic expression for $\hat{v}$. These expressions are solved and plotted in Figure 2 with $\epsilon_{\mathrm{x}}$ set to 0.3 .

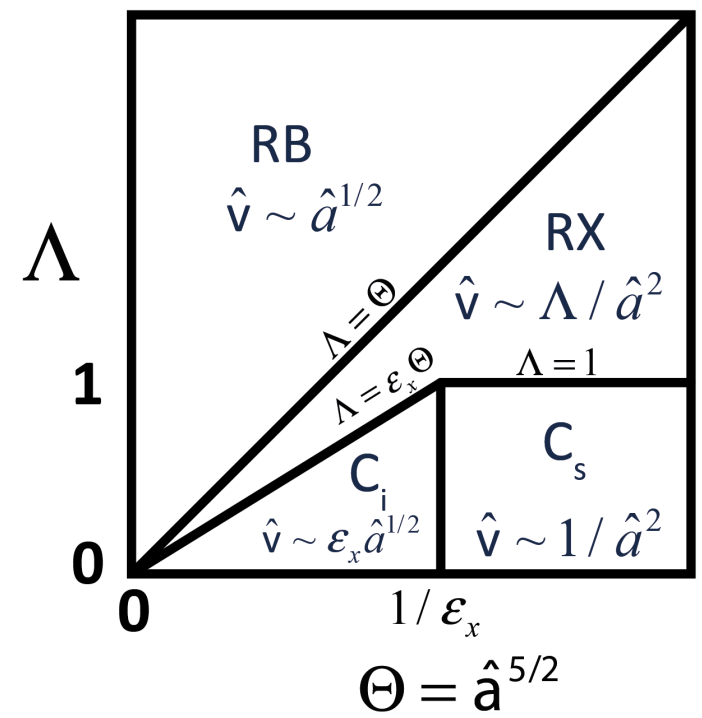

Figure 1 The $\Lambda-\Theta$ regime diagram with the normalized velocity scalings for each regime. Reproduced from [J. R. Myra, D. A. Russell, and D. A. D'Ippolito, Phys. Plasmas 13, 112502 (2006)], with the permission of AIP Publishing.

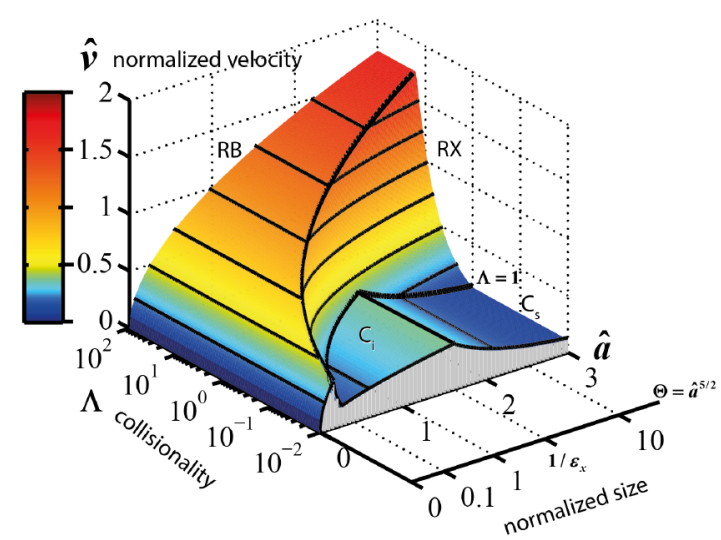

Figure 2 (Color online) The normalized velocity $\hat{v}$ solved for relevant ranges of $\hat{a}$ and $\Lambda$, and with $\epsilon_{\mathrm{x}}=0.3$, which is a typical value for diverted plasma configurations.

The different regimes are classified as follows:

- $\mathrm{C}_{\mathrm{s}}$ : sheath-connected regime

- At sufficiently low collisionality $(\Lambda<1)$, large filaments extend all the way to the outer target, and the dominant return current flows through the sheath and is limited by sheath resistivity $[27,28]$.

$$
\hat{v} \sim 1 / \hat{a}^{2}
$$

- $\mathrm{C}_{\mathrm{i}}$ : connected ideal-interchange regime

- These filaments are small enough that the ion polarization drift is the dominant return current path. Elliptical distortion due to magnetic shear is able to compress the filament, shortening the cross-field path of the return current, slowing the filaments $[32,38]$.

$$
\hat{v} \sim \varepsilon_{x} \hat{a}^{1 / 2}
$$

- RX: Resistive x-point regime

- In this regime, the dominant return current path is the cross-field resistivity, which is magnified by the strong squeezing of the magnetic flux tubes near the X-point due to magnetic shear and poloidal flux expansion[75]. The magnetic shear acts to compress the filament, shortening the return current path[76]. The parallel current is limited by the plasma resistivity $\eta_{\|}$ (represented by the collisionality $\Lambda$ ). This is the only regime where the velocity has a direct dependence on $\Lambda$. 


$$
\hat{v} \sim \Lambda / \hat{a}^{2}
$$

- RB: Resistive Ballooning regime

- When the collisionality is sufficiently high $(\Lambda>\Theta)$ the filaments are not electrically connected to the divertor region. Therefore, the ion polarization drift is the dominant return current path $[38,77]$. The magnetic distortion is assumed to be negligible since the filaments do not extend past the X-point if one exists.

$$
\hat{v} \sim \hat{a}^{1 / 2}
$$

It is important to be aware that these velocity scalings are analytical approximations, utilize asymptotic expressions and were developed by approximating gradients as inverse scale lengths. As such, the scalings and the normalized quantities have uncertainties greater than order unity[38], though seeded filament simulations[32,78,79] and experiments in magnetized torus devices[47,72] suggest that the uncertainties may be smaller.

\section{Experimental Setup}

Due to the stochastic nature of the filaments, a large sample size is needed to provide a statistically relevant comparison between experimental data and theory. To this end, a large database of conditionally averaged samples has been assembled from data taken in the SOL from density scans in both forward and reverse $\mathrm{Bt}$, in diverted and inner wall limited (IWL) configurations, with collisionality varying from the sheath-limited to detached regimes. Databases of similar size have been studied in TORPEX[47,72], NSTX [73,80], and TCV[81]. Filaments measured inside the Last Closed Flux Surface (LCFS) have been omitted from the database since the analytic models discussed are only relevant in the SOL.

Data was taken from 18 ohmic L-mode IWL plasma discharges across a range of line-averaged densities with $1 \mathrm{e} 19 \mathrm{~m}^{-3}<\mathrm{n}_{\mathrm{e}}<6 \mathrm{e} 19 \mathrm{~m}^{-3}$, plasma current $85 \mathrm{kA}$ $<\left|\mathrm{I}_{\mathrm{p}}\right|<210 \mathrm{kA}, \mathrm{B}_{\mathrm{t}}= \pm 1.4 \mathrm{~T}$ (forward and reverse field), ellipticity $1.2<\kappa<1.5$, and with triangularity $\delta=0$. Approximately 600 conditional averaged entries exist for these discharges. The discharges were described in $[82,83]$, and an example equilibrium is shown in Figure $3 a$ ).

Approximately 1200 conditionally averaged data entries were taken in 69 ohmic L-mode lower single null discharges with $2.3 \mathrm{e} 19 \mathrm{~m}-3<\mathrm{n}_{\mathrm{e}}<1.5 \mathrm{e} 20 \mathrm{~m}-3$ (ranging from conduction limited to detached conditions), $240 \mathrm{kA}<|\mathrm{Ip}|<360 \mathrm{kA}, \mathrm{B}_{\mathrm{t}}= \pm 1.4 \mathrm{~T}$ (forward and reverse field), and with divertor flux expansion factors $2.1<f_{\text {exp }}<14$ as shown in Figure $3 b)$ and c). The discharge conditions were described in detail in [67,84-86]. Included in these discharges was an $f_{\exp }$ scan in order to manipulate $\mathrm{L}_{\|}^{\mathrm{x}-\mathrm{pt}}$ within the divertor. This allowed the divertor conditions (including $\Lambda_{\text {div }}$ ) to be modified with minimal impact on the upstream conditions. If the dominant return current path occurs in the divertor, as is described for the $\mathrm{C}_{\mathrm{i}}, \mathrm{C}_{\mathrm{s}}$ and $\mathrm{RX}$ regimes, then significant changes in filament behavior should be observed by modifying $f_{\text {exp }}$ alone. This hypothesis was tested in ref [67], and it was found that the scan in $f_{\exp }$ did not affect filament size, filament radial velocity or the upstream density profile in TCV.

The principal measurements were made using a fast reciprocating probe mounted at the midplane of TCV [87]. The location of the probe is shown in Figure 3. The background $\mathrm{T}_{\mathrm{e}}$ and $\mathrm{n}_{\mathrm{e}}$ measurements were taken using the double probe technique while accounting for Debye sheath expansion using the Perimeter sheath expansion method[88]. These measurements range between $5 \mathrm{eV}<\mathrm{T}_{\mathrm{e}}<60 \mathrm{eV}$ and $1 \mathrm{e} 18 \mathrm{~m}^{-3}<\mathrm{n}_{\mathrm{e}}<$ $6 \mathrm{e} 19 \mathrm{~m}^{-3}$ throughout the database.
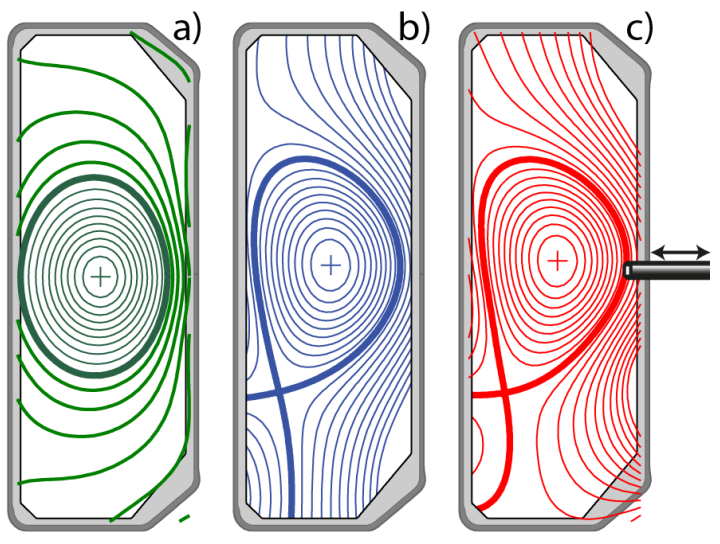

Figure 3 Example equilibria for (a) an inner wall limited plasma, (b) a lower single null with small flux expansion at the outer target and (c) a lower single null with large flux expansion and flux flaring in the outer target. The reciprocating probe enters horizontally at the OMP. 

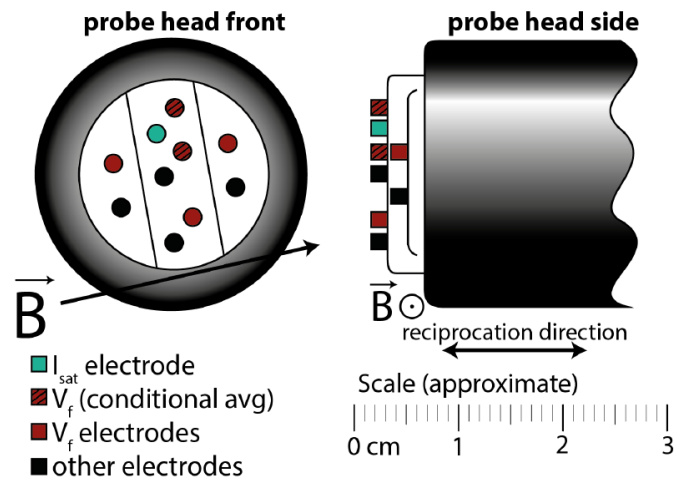

Figure 4 The reciprocating probe head electrode layout. Two $V_{f}$ electrodes are bracketing the $\mathrm{I}_{\text {sat }}$ electrode, allowing for the determination of $E_{\theta}$ for events centered over the three electrodes.

An electrode measuring the ion saturation current $\mathrm{I}_{\text {sat }}$ is straddled by two electrodes measuring the floating potential $\mathrm{V}_{\mathrm{f}}$ (see Figure 4). The $\mathrm{V}_{\mathrm{f}}$ electrodes provide the local poloidal electric field $\mathrm{E}_{\theta}$ and radial drift velocity $\mathrm{V}_{\mathrm{r}}$ centered over the $\mathrm{I}_{\text {sat }}$ electrode (assuming that $T_{e}$ is constant over the three electrodes). $E_{\theta}$ and $\mathrm{V}_{\mathrm{r}}$ are zero on average, so net radial transport only occurs if the fluctuations in $\mathrm{V}_{\mathrm{r}}$ are in phase with the fluctuations in $n_{e}$. In order to determine the radial velocity for the filaments, they need to be isolated from the broadband turbulence. This was accomplished using the conditional averaging procedures described in [25] for the general case, and in [29] for a similar electrode geometry.

All signals were first filtered with a bandpass between $12 \mathrm{kHz}$ and the Nyquist frequency. The low cutoff frequency is just above the $10 \mathrm{kHz}$ typical switching frequency of the fast power supply that drives TCV's vertical stabilization coils[89]. This removes the contributions from $\mathrm{MHD}$, oscillations in the plasma position, the motion of the reciprocating probe, as well as the background non-fluctuating components before conditional averaging. $\left|\delta \mathrm{I}_{\text {sat }} / \mathrm{I}_{\text {sat }}\right|$ was typically $\sim 50 \%$ with a skewness $\sim 0$ near the LCFS and increasing to 2-3 in the far-SOL.

Peaks in $\mathrm{I}_{\text {sat }}$ exceeding +2.5 standard deviations $(\sigma)$ were sampled from $2 \mathrm{~ms}$ windows of data. The peaks were aligned and averaged along with the surrounding $100 \mu$ s of $\mathrm{I}_{\text {sat }}$ and $\mathrm{E}_{\theta}$ data, see Figure 5.

Simulations show that filaments feature a rapidly advecting cell and a density wake which is left behind (see e.g. [40,78,90]). This trailing wake does not advect with the same velocity as the rest of the filament. This can be seen in the example shown in Figure 5. The $\mathrm{I}_{\text {sat }}$ trace remains elevated above average after the peak has passed $(+10$ to $+20 \mu \mathrm{s})$, which is long after the $\mathrm{E}_{\theta}$ trace (and thus, the radial velocity) has returned to zero. The poloidal velocity was also found to be near zero in this example (methodology discussed below) suggesting that the decrease in $I_{\text {sat }}$ which occurs after $t=5 \mu$ s is due to diffusion rather than advection. The filament equivalent circuit model discussed above only pertains to the strongly polarized high-density region. Therefore, in this study, we will define our filament width to ignore these trailing wakes. The filament temporal width $\tau_{\mathrm{FWHM}}$ was taken by fitting a Gaussian centered at the $\mathrm{I}_{\text {sat }}$ peak (shown in black), with the fit weighted preferentially towards the steep front edge of the signal. The filament's $E_{\theta}$ was taken at the time of the peak in $\mathrm{I}_{\mathrm{sat}}$, and the radial velocity was calculated using $v_{r}=\left(\vec{E}_{\theta} \times \vec{B}_{t}\right) / B^{2}$. We find that these

trailing wakes can increase the measured $\tau_{\mathrm{FWHM}}$ by up to a factor of 2 if they are not ignored.

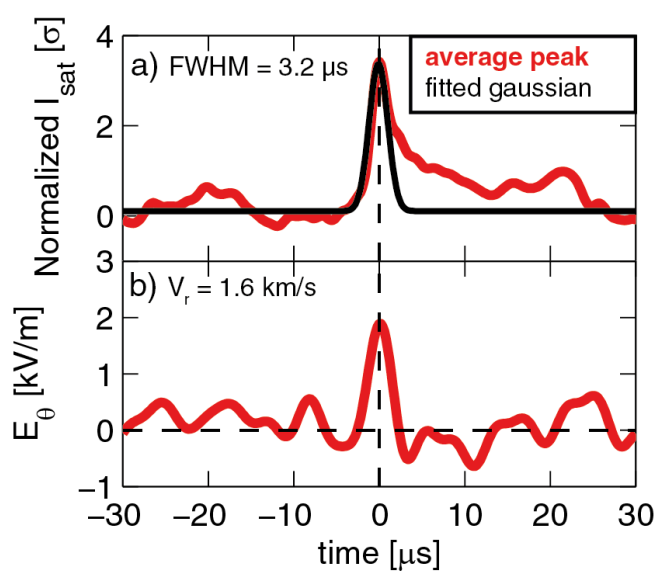

Figure 5 An example of the conditional averaging results from $2 \mathrm{~ms}$ of data. The blob width was determined by fitting a Gaussian to the normalized $\mathrm{I}_{\mathrm{sat}}(\mathrm{a})$. $\mathrm{E}_{\theta}$ (b) for the blob is taken from the averaged signal at time $=0$.

In order to calculate the size of the filament, an estimate of the poloidal velocity $\mathrm{V}_{\text {pol }}$ is also needed. This was determined by assuming that the filaments are entrained in the poloidal flows, the velocity of which was determined using the cross-correlation method $[29,91,92]$ which was applied to $2 \mathrm{~ms}$ timeslices of $V_{f}$ data. However, it was found that the poloidal velocity could be greatly over-estimated when using 1D time delay cross-correlation methods since the 1D method has a non-unique solution (See $[93,94]$ for more details). This was especially true where $\mathrm{V}_{\text {pol }}$ is small, which is typically true in the farSOL, and in the center of the shear layer. Therefore, the poloidal flow velocity was determined using 2D cross-correlation $[93,94]$ using all $5 \mathrm{~V}_{\mathrm{f}}$ electrodes 
identified in Figure 4. This results in $\mathrm{V}_{\text {pol }}$ values agree with the force balance equation[95], which dictates that the radial electric field must be balanced against the poloidal ion diamagnetic drift and the poloidal rotation velocity. This will be shown in section 3. Compared to the 1D method, 2D crosscorrelation was observed to reduce the $\mathrm{V}_{\text {pol }}$ by as much as a factor of 5 .

The filament radius is then calculated by assuming a circular cross-section, since we lack the ability to measure the shape of the filaments.

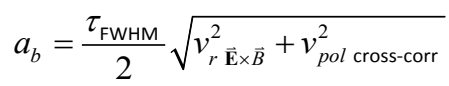

We find that ignoring the trailing wake and using 2D cross-correlation significantly reduces the size estimates for large filaments but has relatively little effect $(<20 \%)$ for the filaments which were already smaller than $1 \mathrm{~cm}$ in diameter. As a result, the filament sizes reported are all smaller than or approximately equal to the outer wall gap. Without these improvements, many filaments were larger than the outer wall gap, a result which has been shown in other conditional averaging studies[14,15].

We will now attempt to quantify the experimental uncertainties for the conditionally averaged sizes and radial velocities in the database. The largest uncertainty for the determination of $a_{b}$ likely stems from the fact that we cannot infer the shape of the filaments. Filament velocity depends chiefly on the vertical size of the filament $\left(\mathrm{L}_{\mathrm{pol}}\right)$ rather than the radial size $\left(\mathrm{L}_{\mathrm{rad}}\right)$. The circular cross-section assumption is supported by early 2D Beam Emission Spectroscopy videos of filaments[29] and fluid modeling[28] which shows that filaments can be elongated near the LCFS where Reynolds stress is high, but that the internal rotation tends to cause the filaments to return to a circular cross-section and maintain that shape (on average) through most of the SOL. However, a recent and more detailed gas puff imaging surveys at NSTX report an average height to width ratio of $\mathrm{L}_{\mathrm{po}} / \mathrm{L}_{\mathrm{rad}}=1.5$ across the whole $\mathrm{SOL}$ for ohmic plasmas[73]. We can estimate a reasonable uncertainty in $\mathrm{a}_{\mathrm{b}}$ for TCV by assuming an even distribution of the velocity angle $\tan \left(\mathrm{v}_{\mathrm{r}} / \mathrm{v}_{\mathrm{pol}}\right)$ for an elliptical filament with $\mathrm{L}_{\mathrm{po}} / \mathrm{L}_{\mathrm{rad}}=1.5$. This would result in a systematic underestimation of ab by $29 \%$ with a random uncertainty of $18 \%(1 \sigma)$. The systematic uncertainty will not affect the conclusions of this paper, but we will consider a random uncertainty of $\sim 20 \%$ when considering the scatter in the measurements presented in sections 3 and 4 .
The uncertainty in $\mathrm{V}_{\mathrm{r}}$ was estimated by comparing the velocities calculated using the ExB method described above and by using 1D cross-correlation of the conditionally averaged signals between the radially separated $V_{f}$ electrodes shown in Figure 4. The two methods agreed well in sign and magnitude with an error distribution of $\pm 0.3 \mathrm{~km} / \mathrm{s}(1 \sigma)$.

One also needs to consider whether the filaments or the background plasma are perturbed by the presence of the probe. Perturbation of $\mathrm{T}_{\mathrm{e}}$ and $\mathrm{n}_{\mathrm{e}}$ within the filaments by the presence of a solid limiter such as the probe head should be negligible since these perturbative effects should occur on the timescale of the SOL particle dwell time $\left(\tau_{\mathrm{SOL}} \sim \mathrm{L}_{\|} / \mathrm{c}_{\mathrm{s}} \sim 300 \mu\right.$ s for TCV). Meanwhile, $v_{p o l}$ and $v_{r}$ are on the order of $1 \mathrm{~km} / \mathrm{s}$ (verified in section 3) such that filaments traverse the probe-head poloidally ( $\mathrm{w}_{\text {probe }} \cong 2.5 \mathrm{~cm}$ wide) or the width of the SOL radially ( $\mathrm{wsL}_{\mathrm{SOL}}=3-5 \mathrm{~cm}$ ) in $(\mathrm{w} / \mathrm{v} \cong 30 \mu \mathrm{s})$ or in about $0.1 \tau_{\mathrm{sol}}$. The background plasma should also experience little perturbation (at least from the sink of plasma particles to the probe head) since it also rotates at $\mathrm{v}_{\text {pol, }}$, such that the flux tubes advect past the probe head in $\sim 0.1 \tau_{\text {sol }}$. This suggests that the presheath "shadow" does not have time to reach a steady-state particle balance and does not therefore need to be 'filled in' by enhanced crossfield fluxes as suggested in [96]. However, the nonperturbative hypothesis has yet to be demonstrated experimentally or by detailed modeling.

Perturbative effects of the probe head to $V_{f}, E_{\theta}$, and $v_{r}$ are more difficult to quantify, but radial velocities, filament sizes, and the skewness of the density signals measured with reciprocating probes were found to agree with those measured by nonperturbative diagnostics including Beam Emission Spectroscopy[29] and by Gas Puff Imaging[97] suggesting that these effects are small.

$\mathrm{T}_{\mathrm{e}}$ is also known to fluctuate in the SOL and the peaks in $\mathrm{T}_{\mathrm{e}}$ have been shown to correlate with the peaks in $\mathrm{I}_{\text {sat }}[29,31,98]$ demonstrating that filaments often have a higher temperature than the surrounding plasma.. Our estimates of $E_{\theta}$, and $v_{r}$, assume that $T_{e}$ is the same at the two $\mathrm{V}_{\mathrm{f}}$ electrodes, which should be a reasonable approximation if 1 ) the conditional averaging method selects for filaments that pass with their centers directly over the $I_{\text {sat }}$ tip, and 2) if $T_{e}$ is peaked symmetrically about the center of the averaged filaments. We believe therefore that the $T_{e}$ fluctuations do not have a direct effect on $v_{r}$ measurements.

The filamentary transport models being evaluated assume that $T_{e}$ is constant and are known to be 
inaccurate when the filament temperature exceeds a critical ratio:[37]

$$
\left(\frac{\Delta T}{T_{b g}}\right)_{c r i t} \equiv\left(\frac{\rho_{s} L_{\|}^{3}}{R^{4}}\right)^{1 / 5}
$$

Where $\Delta \mathrm{T}$ is the change in temperature above the background temperature $\mathrm{T}_{\mathrm{bg}}$. The right-hand side of the definition evaluates to $\sim 0.9$ for the conditions considered in this paper. The elevated filament temperature causes the filament to contain a higher electric potential than the surrounding plasma, and the radial electric field causes the filaments to rotate[48]. Above the critical temperature ratio, the filament spins sufficiently quickly to reduce the vertical charge separation and can greatly reduce the radial velocity[48]. $\Delta \mathrm{T} / \mathrm{T}_{\mathrm{bg}}$ has been measured to exceed $0.9[98]$, and one conditional averaging example has been shown with $\Delta \mathrm{T} / \mathrm{T}_{\mathrm{bg}} \sim 2[29]$. Unfortunately, we lack the ability to measure $T_{e}$ within the filaments. The ramifications of this and other known processes which are not included in the velocity scaling (described in section 1) will be discussed in section 4 , while the size and velocity distributions are discussed in the next section.

\section{Size and Velocity Distribution}

Electrostatic simulations for filaments predict that filaments are most stable at an optimal size of $\hat{a}=$ $1[40]$ since smaller filaments $(\hat{a}<1)$ are vulnerable to the Kelvin-Helmholtz instability[77], and larger filaments $(\hat{a}>1)$ are vulnerable to curvature-driven instabilities[34]. It is expected therefore that the filaments should be observed to cluster around this optimal size. This is a useful point of comparison between experimental data and theory [99].

The size distribution is shown in Figure 6 for the IWL discharges and in Figure 7 for the lower single null discharges with typical outer wall gaps shown by the dashed lines. Filaments which are smaller than the $4 \mathrm{~mm}$ distance between the electrodes used to measure $E_{\theta}$ are shaded in red. Since the $E_{\theta}$ measurement may be underestimated, $\mathrm{v}_{\mathrm{r}}$ and thus $\mathrm{a}_{\mathrm{b}}$ could also be underestimated for those data points. In both cases, the population distribution has a peak FWHM diameter $2 \mathrm{a}_{\mathrm{b}} \sim 5 \mathrm{~mm}$ (Figure $6 \mathrm{a} \&$ Figure $7 \mathrm{a}$ ), and $\hat{a}$ peaks between 0.3 and 0.5 for the limited discharges (Figure 6b), and between 0.5 and 0.8 for the diverted discharges (Figure 7b).

To check if the conditional averaging method was biasing the sample population towards larger filaments, the sampling threshold was lowered from
$2.5 \sigma$ down to $2.1 \sigma$. This did not change the shape or peak of the normalized size distribution. The number of collected filaments approximately doubles, but new events are added evenly across the size distribution, maintaining the peak at $\hat{a} \sim 0.5$. The sampling threshold was therefore kept constant at $2.5 \sigma$ for all conditions.

The multi-machine database in ref [99] shows a distribution of $1.5<\hat{a}<15$ and $0.5 \mathrm{~cm}<\mathrm{a}_{\mathrm{b}}<4 \mathrm{~cm}$ as measured by a wide range of techniques, so the filaments in TCV are considerably smaller in normalized size than those typically reported in other tokamaks. The definition of the filament width used in this paper contributes but is not sufficient to explain the difference in size. In section 4, we will discuss the ramifications of the small filament sizes, which may explain why some of the density shoulder evolution trends appear to be weak in TCV.

Next, we will consider the radial velocities of the filaments. The radial velocity distribution for the IWL plasmas is shown in Figure 8 where the velocities range from $0-3 \mathrm{~km} / \mathrm{s}$ and where most filaments have velocities clustered between 0.5 and $1.5 \mathrm{~km} / \mathrm{s}$. The radial velocity distribution for the diverted plasmas is shown in Figure 9 where most filaments have velocities clustered between 0.5 and 2 $\mathrm{km} / \mathrm{s}$, with a total range between $-4 \mathrm{~km} / \mathrm{s}$ to $+5 \mathrm{~km} / \mathrm{s}$.

Filaments with negative velocity have been observed with GPI before [80], but the negative velocities have much smaller magnitudes than the positive velocities. To our knowledge, this is the first experimental evidence of rapid inward filamentary convection. The inward moving filaments were only found in diverted plasmas in reverse field $(\mathbf{B} \times \nabla \mathbf{B}$ away from the $\mathrm{X}$ point) close to the LCFS. An example is shown in Figure 10a) where the radial velocities are plotted as a function of the distance from the separatrix (R$R_{\text {sep}}$ ). The radial velocities are positive for the majority of the SOL and are negative for R- $\mathrm{R}_{\text {sep }}<0.5$ $\mathrm{cm}$. At the same radii, the poloidal velocity as measured using 2D cross-correlation (see Figure 10b) also changes rapidly. We find that the largest inward velocity measurements typically occur in the region of highest poloidal velocity shear. Note that we are only considering the conditionally averaged radial velocities for positive $\mathrm{I}_{\text {sat }}$ peaks, so the radial velocities of density holes do not contribute to the values shown here.

The thick red line in Figure 10b) is the expected poloidal rotation velocity calculated using the singleion force-balance equation[95], with the $\mathrm{E}_{\mathrm{r}} \mathrm{xB}_{\phi}$ component shown by the blue line. 


$$
V_{p o l}=\frac{\vec{E} \times \vec{B}}{B^{2}}-\frac{\nabla p_{i} \times \vec{B}}{q_{i} n B^{2}}
$$

Where $\mathrm{p}_{\mathrm{i}}$ is the ion pressure and $\mathrm{q}_{\mathrm{i}}$ the charge of the ion. $V_{\text {pol }}$ was calculated from the background $T_{e}, n_{e}$, and $\mathrm{V}_{\mathrm{f}}$ measurements from the double probe, assuming that the ion temperature $\mathrm{T}_{\mathrm{i}}=\mathrm{T}_{\mathrm{e}}$ and that the plasma potential $\mathrm{V}_{\mathrm{p}}=\mathrm{V}_{\mathrm{f}}+2.5 \mathrm{kT}_{\mathrm{e}}$. The agreement between the poloidal velocities and the force balance equation line suggests that the $2 \mathrm{D}$ cross-correlation method is accurate.

At this time, we are unaware of any theories which may explain the rapid inward velocities observed. SOLT simulations occasionally show inward motion of some filaments which are caught in the wake of much larger filaments [100], but the magnitude of the inward velocities are a small fraction of the outward velocities. Regions of diamagnetic plasma are known to exist at the plasma edge that can attract filaments since they are also diamagnetic [58]. This effect only takes place on closed field lines, which could explain why inward moving filaments are not observed in the far-SOL. However, this effect is likely too small to explain the magnitude of the velocities observed. Finally, simulations show that filaments within a poloidal velocity shear layer can become trapped, with their radial velocity tending towards zero[101], so it is known that the shear layer can affect the radial velocities of filaments. Since our measurements of inward advecting filaments only occur in regions of high poloidal velocity shear, it is likely that this trapping effect plays a role, but it is unclear how they would cause inward motion.
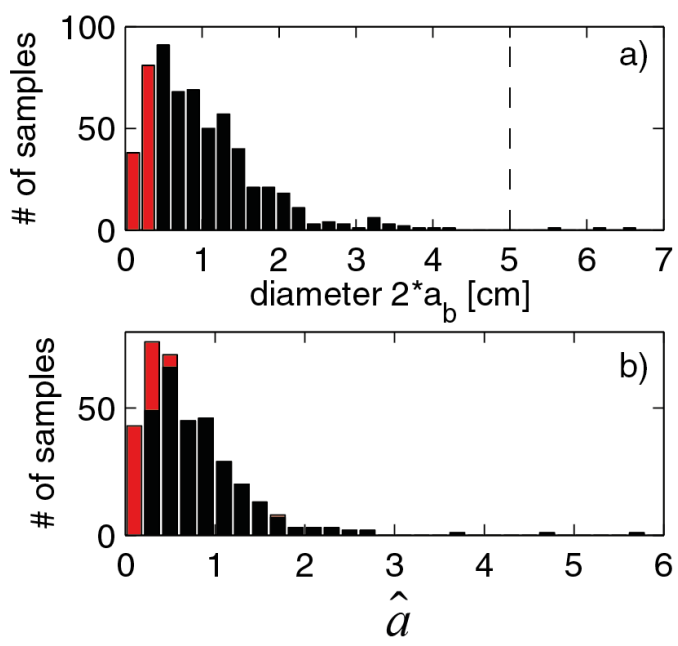

Figure 6 A histogram showing the distribution of the calculated filament diameters (a) and the normalized filament radii (b) for inner-wall limited discharges. The sample population decreases rapidly with increasing size. Filaments with a diameter below $4 \mathrm{~mm}$ are shown in red. The dashed line in (a) indicates the typical gap between the LCFS and the outer wall. The uncertainty for $a_{b}$ is estimated to be $\pm 20 \%(1 \sigma)$.

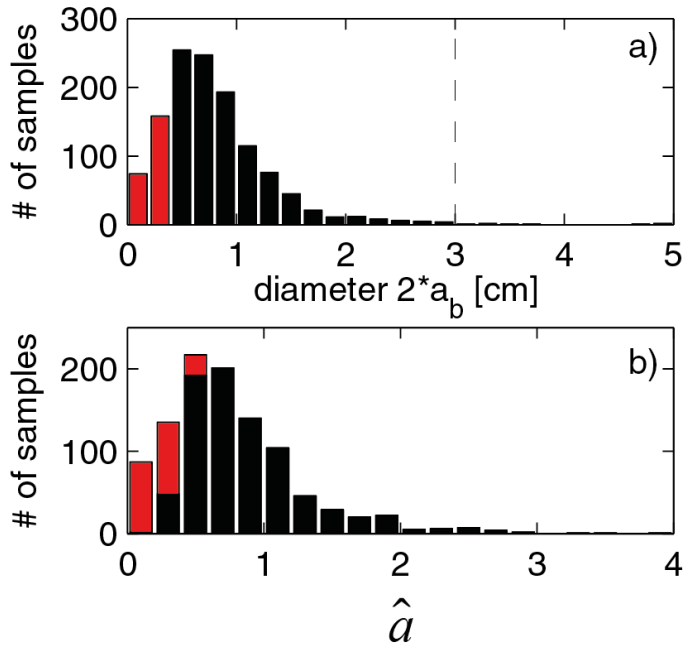

Figure 7 A histogram showing the distribution of the calculated filament diameters (a) and the normalized filament radius (b) for lower single null discharges. The sample population decreases rapidly with increasing size. Filaments with a diameter below $4 \mathrm{~mm}$ are shaded red. The dashed line in (a) indicates the typical gap between the LCFS and the outer wall. The uncertainty for $a_{b}$ is estimated to be $\pm 20 \%(1 \sigma)$. 


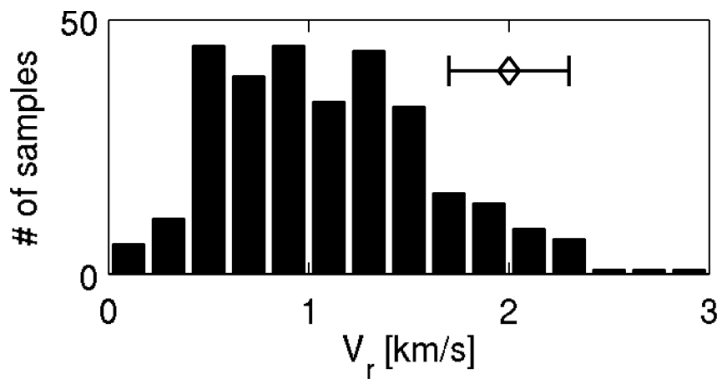

Figure $8 \mathrm{~A}$ histogram showing the distribution of radial velocities for inner-wall limited discharges. The horizontal error-bars represent the typical measurement uncertainty $( \pm 1 \sigma)$.

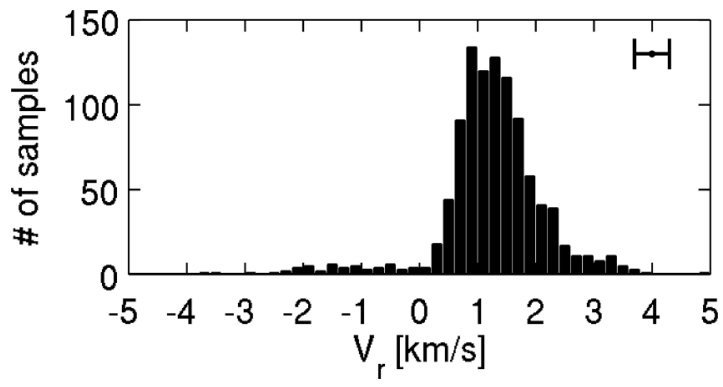

Figure 9 A histogram showing the radial velocity distribution for the lower single null discharges. A small population of filaments was found to be moving inward. The horizontal error-bars represent the typical measurement uncertainty $( \pm 1 \sigma)$.

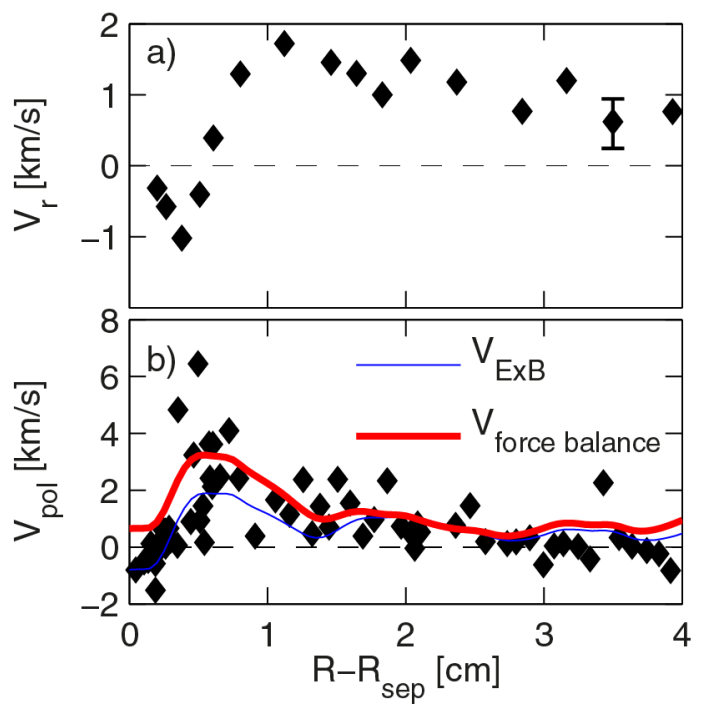

Figure 10 The radial velocities for conditional averaged measurements are shown in (a) and the poloidal velocity determined by $2 \mathrm{D}$ cross-correlation are shown in (b) as a function of the distance from the separatrix $\left(\mathrm{R}-\mathrm{R}_{\mathrm{sep}}\right)$. The thick red line in (b) is the expected rotation velocity calculated from the force balance equation. The blue line shows the $\mathrm{E}_{\mathrm{r}} \mathrm{xB}_{\phi}$ contribution. The estimated uncertainty in $V_{r}$ is shown by the example errorbars $(1 \sigma)$, while the uncertainty in $V_{\mathrm{pol}}$ is represented by the scatter.

\section{Velocity Scaling Validation}

We now proceed to investigate the velocity scaling itself, starting by sorting the database entries throughout the collisionality-size $\Lambda-\hat{a}$ space. Figure 11 shows the filament distribution (black dots) throughout the $\Lambda_{\text {mid- }} \hat{a}$ space for the IWL plasmas. For these discharges, the glancing field angle with the flush mounted wall Langmuir probes prevents accurate determination of $n_{e}$ at the target, so we will not consider $\Lambda_{\text {div. }}$. The red 'o's represent filaments which are smaller than the separation between the $\mathrm{E}_{\theta}$ electrodes. The magnetic distortion factor $\epsilon_{\mathrm{x}}$ was set to 0.5 based on a typical poloidal flux expansion at the top and bottom of the plasma. Note that the filaments predominately populate the ResistiveBallooning RB regime in the $\Lambda_{\text {mid }} \hat{a}$ space (66\% of the black points).

For diverted plasmas, recent work $[14,15]$ suggests that the collisionality in the divertor $\Lambda_{\text {div }}$ is more important than $\Lambda_{\text {mid. }}$. This should be especially true if the transition between the sheath connected and sheath disconnected regimes is important since $\Lambda_{\text {div }}$ is meant to represent the high collisionality region just in front of the target which serves as the final barrier preventing the filaments from connecting to the target.

The filament distribution throughout the $\Lambda_{\text {div }} \hat{a}$ space for the diverted plasmas is plotted in Figure 12. As above, the red 'o's show filaments with diameters $<4 \mathrm{~mm}$. $\epsilon_{\mathrm{x}}$ was taken as 0.3 . The approximate values for $\Lambda_{\text {mid }}$ are shown on the right axis. Once again, the filaments are predominately found to populate the RB regime, which contains $\sim 80 \%$ of the black points, or $\sim 90 \%$ if one uses $\Lambda_{\text {mid }}$ instead of $\Lambda_{\text {div }}$.

The small average size of the filaments means that very few populate the regions of the $\Lambda_{\text {div }}-\hat{a}$ space where $\hat{v}$ depends most strongly on collisionality (The part of the RX regime with $\hat{a}>1.6, \Theta>3.3$ ). $\hat{v}$ should only change very modestly as a function of $\Lambda$ for small filaments, and $\hat{v}$ is predicted to be constant with $\Lambda$ in the RB regime (see Figure 2). This likely explains why modifying the collisionality was not found to have a straightforward relationship to the upstream density profiles in TCV [67]. 


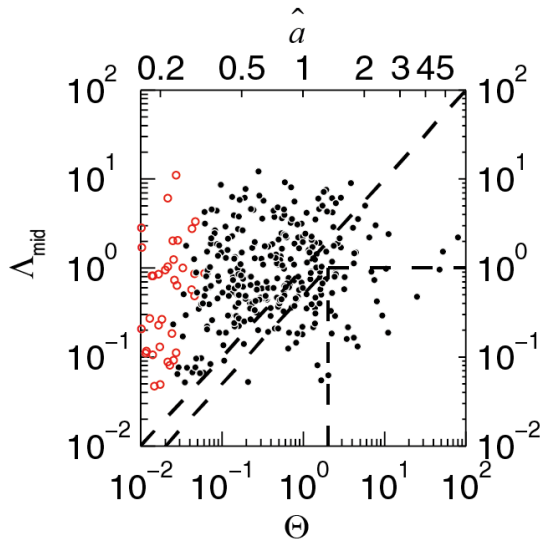

Figure 11 The filaments size and collisionality distribution for the inner wall limited discharges. Each conditional averaged element is represented by a black point. Red 'o's have a calculated diameter $<$ $4 \mathrm{~mm}$.

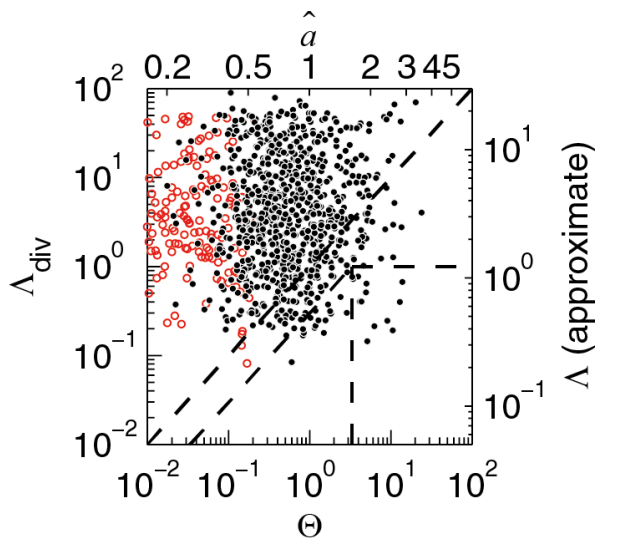

Figure 12 The filaments size and collisionality distribution for the lower single null discharges. Each conditional averaged element is represented by a black point. Red 'o's have a calculated diameter < $4 \mathrm{~mm}$.

\subsection{Velocity Scaling in the Resistive Ballooning Regime}

The sample size is large enough to consider an experimental validation of the RB velocity scaling for both the IWL discharges and the diverted discharges. A velocity scaling comparison for the IWL discharges is shown in Figure 13, where we plot only the database entries found to populate the RB regime in Figure 11. The shaded region identifies the filaments which are smaller than the separation between the $\mathrm{E}_{\theta}$ electrodes. The measured radial velocities show a scatter which is larger than the experimental uncertainty and is approximately bounded by the RB velocity scaling eq (1.9) with $\hat{v} \sim \hat{a}^{1 / 2+0.2}$ (this notation signifies that $\hat{v}$ ranges from $\hat{a}^{1 / 2}-0.6$ up to $\hat{a}^{1 / 2}+0.2$ ). The experimental uncertainties were assessed to be $\sim 50 \%$ for $\mathrm{a}_{\mathrm{b}}$ and $\sim 20 \%$ for $\mathrm{v}_{\mathrm{r}}$ in section 2 .

Low $V_{\mathrm{r}}$ measurements exist for all values of $\hat{a}$. These measurements are consistent with the fact that the scalings estimate the polarization attainable for the idealized filament, while mechanisms exist which can prevent individual filaments from reaching this electric field strength. As discussed in section 1, most of these interactions act to limit the vertical polarization of the filaments and to decrease their radial velocity. Elevated temperatures within the filaments, in particular, could drastically reduce the radial velocities below the scaling.

The magnitude of the scatter around and below the theoretical scaling is similar to that found using a database of similar size taken from the magnetized torus TORPEX comparing filament velocities against the $\mathrm{C}_{\mathrm{i}}$ and $\mathrm{C}_{\mathrm{s}}$ scalings [72], confirming that the scatter in velocities is intrinsic to the filaments. Therefore, we can conclude that the filament database is consistent with the RB scaling for IWL discharges, but that only a fraction of the filaments are able to reach their ideal polarization and ExB velocity.

The RB velocity scaling comparison for the diverted plasmas is shown in Figure 14a), showing only the database entries in the RB regime as calculated using $\Lambda_{\text {div. }}$. The small population (7\%) of filaments with negative radial velocities appear to roughly follow $\hat{v}=-\hat{a}$. As discussed in section 3, the filaments with negative radial velocities are only measured in the shear layer close to the LCFS. The color of the data points corresponds to the distance from the separatrix $\left(R-R_{\text {sep }}\right)$ in Figure 14a).

The heat map in Figure 14b) shows the density of the data points more effectively than part a). Here, we see that the normalized velocities are clustered predominately in the region $\hat{v} \sim \hat{a}^{1 / 2}{ }_{-0.6}^{+0.6}$, roughly scattered around the RB velocity scaling. Again, we can conclude that the velocities are consistent with the $\mathrm{RB}$ velocity scaling within its expected uncertainty range. These measurements show a higher average radial velocity and a larger scatter than the IWL dataset for any given size. This difference is not predicted by the model, which contains no geometric effects for the filaments in the $\mathrm{RB}$ regime. We can see that the average radial velocity increases with size as predicted, though it is not clear within the scatter if the velocities follow the proportionality of the $\hat{v} \sim \hat{a}^{1 / 2}$ scaling. 


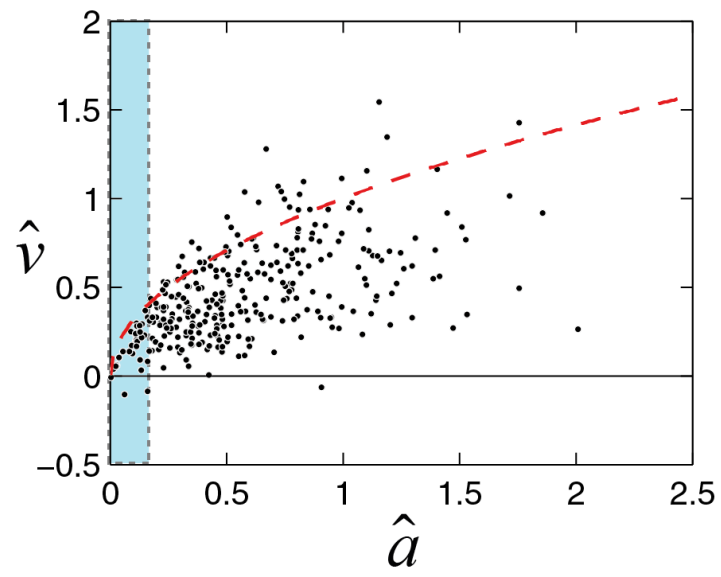

Figure 13 The normalized filament radial velocities as a function of the normalized filament radius for Resistive-Ballooning conditions in IWL discharges. The dashed line is the $\hat{v} \sim \hat{a}^{1 / 2}$ velocity scaling. The shaded region identifies filaments which are smaller than $4 \mathrm{~mm}$ in diameter.
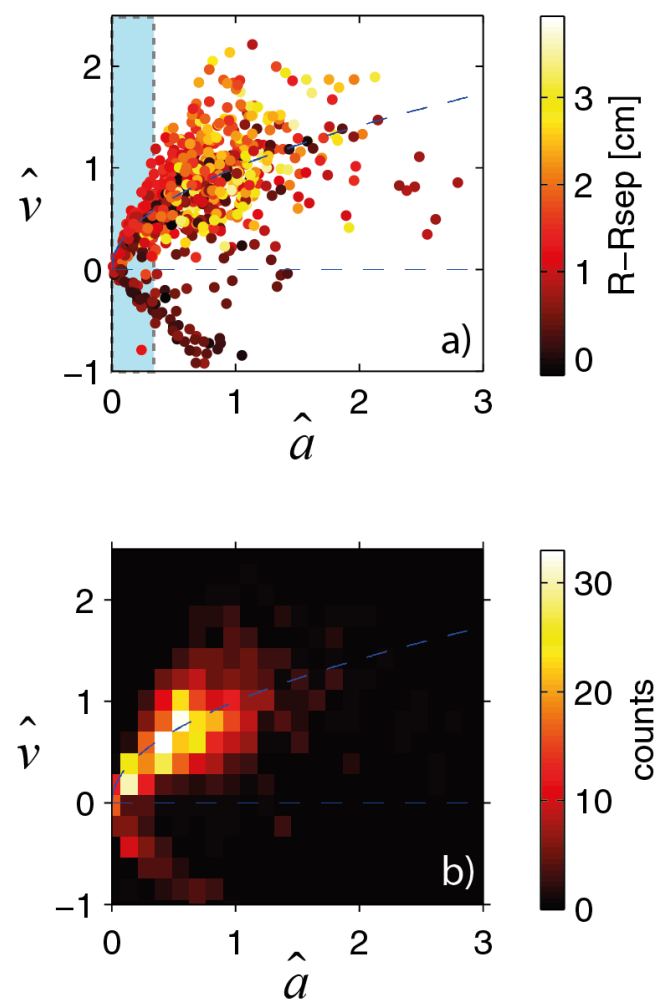

Figure 14(color online) The normalized filament radial velocities as a function of the normalized filament radius for ResistiveBallooning conditions in lower single null discharges. The dashed line represents the $\hat{v} \sim \hat{a}^{1 / 2}$ velocity scaling. The conditionally averaged results are shown by the data points. Part (b) is a heat map of the data in part (a). The shaded region in (a) show filaments which are smaller than $4 \mathrm{~mm}$ in diameter.

\subsection{Velocity Scaling for Large Filaments}

Outside of the RB regime, there are fewer measurements in the database, which are split between the $\mathrm{C}_{\mathrm{i}}, \mathrm{C}_{\mathrm{s}}$, and $\mathrm{RX}$ regimes. This makes it more difficult to evaluate the data against the velocity scaling, but we will present a few useful comparisons in this subsection.

For the IWL discharges, $\hat{v}$ is plotted as a function of $\hat{a}$ in Figure 15 for all the filaments outside of the RB regime. The red dashed line shows the velocity scaling for the $\mathrm{C}_{\mathrm{i}}$ and $\mathrm{C}_{\mathrm{s}}$ regimes, while the blue dotdashed lines show the velocity scaling for $\Lambda=1$ and $\Lambda=2$ (the bottom and top branches respectively) which covers the RX regime. For $\hat{a}>1.8, \hat{v}$ is expected to decrease with size following the $\hat{v} \propto 1 / \hat{a}^{2}$ contours. The dataset does indeed show that $\hat{v}$ decreases as a function of $\hat{a}$ for large values of $\hat{a}$ and shows a peak near $\hat{a}=1$ in agreement with the model. Additionally, this decreasing trend demonstrates that the relationship observed for the $\mathrm{RB}$ regimes in section 4.1 is not an artifact of the way the filament sizes are calculated using $\mathrm{v}_{\mathrm{r}}$.

Next, we will consider the large filaments from the diverted discharges. The $\hat{v} \propto 1 / \hat{a}^{2}$ relation is less clear for this dataset since it lacks the largest $\hat{a} \sim 6$ filaments. Instead, we will consider the relationship between $\hat{v}$ and $\Lambda$ for filaments with $\hat{a}>1 / \epsilon_{\mathrm{x}}{ }^{2 / 5}=1.6$ (equivalent to $\Theta>1 / \epsilon_{\mathrm{x}}=3.3$ ) since filaments in this size range exist in even distribution for the $\mathrm{C}_{\mathrm{s}}, \mathrm{RX}$, and $\mathrm{RB}$ regimes. i.e. the distribution of $\hat{a}$ stays roughly constant as a function of $\Lambda_{\text {div }}$ in Figure 12 in the region where $\hat{a}>1.6$.

$\hat{v}$ is plotted as a function of $\Lambda_{\text {div }}$ in Figure 16a) and as a function of $\Lambda_{\text {mid }}$ in Figure 16b). The red dashed line in both subplots is the velocity scaling for $\hat{a}$ $=1.6, \Theta=3.3$, and the blue dotted line shows the velocity scaling for $\hat{a}=3.3, \Theta=20$, the upper bound in size for the single null database. Since $\Lambda$ varies by 3 orders of magnitude, it dominates over $\hat{a}$ when determining $\hat{v}$ (this can be seen in Figure 2). Because the population density decreases rapidly with filament size, the red dashed line is more applicable to the dataset.

In Figure 16a), $\hat{v}$ increases as expected for $10^{0}<\Lambda_{\text {div }}$ $<10^{1}$, and appears to be constant (as expected) for $\Lambda_{\text {div }}<10^{\circ}$ in the $\mathrm{C}_{\mathrm{s}}$ regime. However, the average velocity in the $\mathrm{C}_{\mathrm{s}}$ regime exceeds the velocity scaling by a factor of 3 or more and $\hat{v}$ changes as a function of $\Lambda_{\text {div }}$ much more weakly than described by the 
scaling in the RX regime. Alternatively, in Figure 16b), $\hat{v}$ is plotted as a function of $\Lambda_{\text {mid, }}$, and $\hat{v}$ agrees more closely with the scaling with $\hat{v} \sim \hat{v}_{\text {scaling }}^{+0.6}{ }_{-0.6}$, which is approximately the same scatter seen in the $\mathrm{RB}$ regime.

This could be because TCV has a very long outer divertor leg relative to the size of the plasma, and a very open divertor compared to other tokamaks. As such, the collisionality in the divertor may not dominate the system as it does in tokamaks with closed divertors. $\Lambda_{\text {div }}$ is meant to represent the region of high collisionality in front of the target, but there is no reason to expect that this region extends to the $\mathrm{x}$ point when the outer leg is so long. This could explain why modification of $\mathrm{L}_{\|}{ }^{\mathrm{x} p \mathrm{pt}}$ was not found to have an effect on the upstream density profiles or on filament sizes and velocities in TCV [67]. In order to fully understand the role of collisionality in TCV, it may be necessary to use an estimate of $\Lambda$ that accounts for parallel gradients in $v_{\text {ei.. }}$ Such 3D filament models are under development[102].

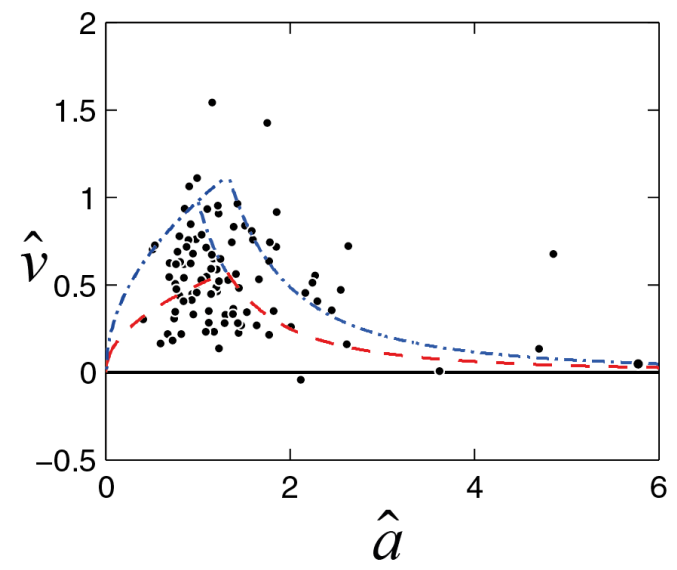

Figure 15 The normalized filament radial velocities as a function of the normalized filament radius for all filaments outside of the $\mathrm{RB}$ regime for the IWL discharges. The red dashed line is the velocity scaling for the $C_{i}$ and $C_{s}$ regimes. The blue dot-dashed lines are the velocity scalings for $\Lambda=1$ (bottom) and $\Lambda=2$ (top).
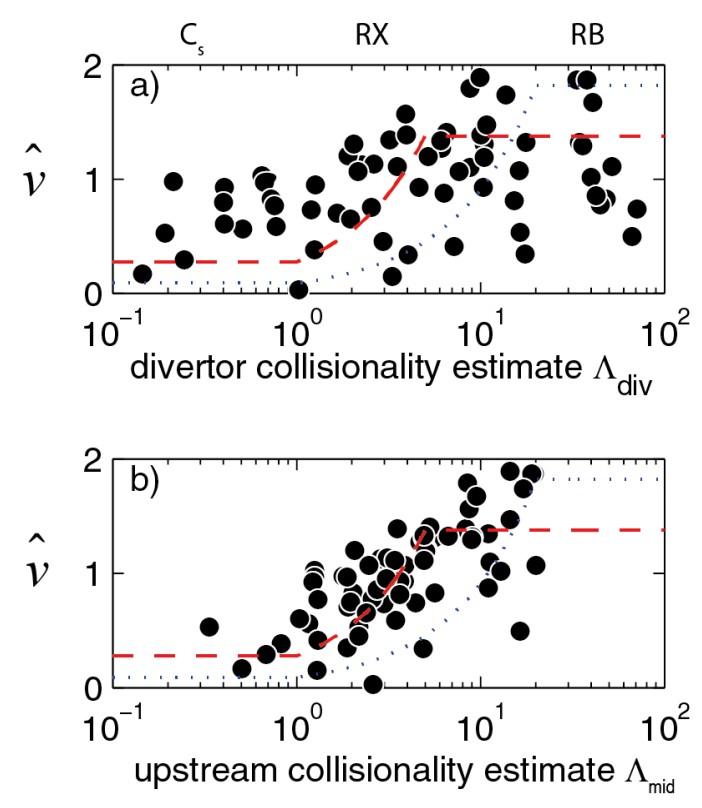

Figure 16 The normalized filament radial velocities as a function of the $\Lambda_{\text {div }}$ (a) and $\Lambda$ (b). The dashed red lines are the velocity scaling for $\hat{a}=1.6$, and the dotted blue lines are the velocity scaling for $\hat{a}=3.3$.

\section{Conclusions}

Using a large database of conditionally averaged measurements in the TCV tokamak, the filament normalized radial velocities $\hat{v}$ were found to be scattered between $\sim 0$ and an upper bound, where this upper bound varies as a function of normalized filament size $\hat{a}$ and the collisionality $\Lambda$ in rough agreement with the velocity scalings. The scatter in the database is larger than the experimental uncertainties, which are estimated to be $\sim 50 \%$ for $\mathrm{a}_{\mathrm{b}}$ or $\hat{a}$ and $\sim 20 \%$ for $\mathrm{v}_{\mathrm{r}}$ or $\hat{v}$. For filaments sorted into the RB regime for IWL plasmas, the upper bound in $\hat{v}$ is confirmed to increase with $\hat{a}$ in agreement with the $\hat{a}^{1 / 2}$ scaling. For filaments in diverted plasmas in the $\mathrm{RB}$ regime, the $\hat{v}$ measurements are higher on average and have a larger scatter than the IWL cases, but $\hat{v}$ is again found to increase with size

approximately in agreement with the $\hat{a}^{1 / 2}$ scaling. These measurements are consistent with the concept that the velocity scaling correctly estimate of the polarization that can be sustained by an ideal filament, but that many mechanisms exist which can act to decrease radial velocity (e.g. filament rotation or filament-filament collisions) or to increase the radial velocity (e.g. filament ellipticity or neutral wind) of any given filament, which randomizes the velocity distribution over a wide range. The role of 
high $T_{e}$ within the filaments compared to the background plasma may be especially important, since it has been shown that $\Delta \mathrm{T} / \mathrm{T}_{\mathrm{bg}}$ can reach sufficiently high ratios to drastically reduce the radial velocity. Future predictive modeling of filamentary transport which use the tested velocity scalings of the filament equivalent circuit model will need to include the statistical spread resulting from these processes, and further study is needed to quantify the magnitude of their effects.

Due to the smaller sample sizes outside of the RB regime, it was more difficult to test the velocity scalings for the other regimes, but the data which is available does appear to be consistent with the velocity scalings. In the IWL discharges for the large filaments in the $\mathrm{RX}$ and $\mathrm{C}_{\mathrm{s}}$ regimes, $\hat{v}$ was found to decrease with filament size which is consistent with the $\hat{v} \propto \hat{a}^{-2}$ velocity scalings. In the diverted discharges for filaments with $\hat{a}>1 / \epsilon_{\mathrm{x}}{ }^{2 / 5}=1.6$ (equivalent to $\Theta>1 / \epsilon_{\mathrm{x}}=3.3$ ) the $\hat{v}$ upper bound has been found to increase with $\Lambda$ in agreement with the velocity scaling of the RX regime. The $\Lambda_{\text {mid }}$ approximation produces a more convincing agreement than $\Lambda_{\text {div }}$, especially in the Cs regime where the $\hat{v}$ measurements exceeded the $\Lambda_{\text {div }}$ scaling by a factor of 3 or more. This could be explained by TCV's open divertor and long outer divertor leg. Due to this geometry, the collisionality in the divertor may not dominate over the main chamber collisionality as it does in tokamaks with closed divertors. Furthermore, the use of $\mathrm{L}_{\|}^{\mathrm{x}-\mathrm{pt}}$ in the definition of $\Lambda_{\text {div }}$ implies that the region of high collisionality just in front of the target extends up to the $\mathrm{x}$-point, which seems inappropriate when $\mathrm{L}_{\|}{ }^{\mathrm{x}-\mathrm{pt}}$ is not small compared to $\mathrm{L}_{\|}$. A more complete approximation for $\Lambda$ (allowing $v_{\mathrm{ei}}$ to change along the field line) and more data at lower collisionality are needed in order to fully understand the role that collisionality plays in TCV.

We find that the majority of filaments populate the $\mathrm{RB}$ regime in TCV. The RB regime contains $66 \%$ of the measured filaments from IWL plasmas and $80 \%$ or $90 \%$ of those from diverted discharges (when sorted using $\Lambda_{\text {div }}$ or $\Lambda_{\text {mid }}$ respectively). This is because the filament in TCV have a size distribution peaking at a diameter of $0.5 \mathrm{~cm}$ and $\hat{a} \sim 0.5$, which is smaller than the 'most stable blob size' expected to have $\hat{a} \sim 1$, though this discrepancy is still within the order unity accuracy that might be expected for the analytic expression for $\hat{a}$. The fact that $80 \%-90 \%$ of the filaments populate the RB regime (where $\hat{v}$ is expected to be independent of $\Lambda$ ) likely explains why the filament radial velocity was not found to increase with line average density in [67]. This also explains why increasing the connection length in the divertor was not found to affect the average filament size or radial velocity since the filaments in the RB regime do not extend into the divertor, as well as why the upstream density profiles were not found to have a straightforward relationship to $\Lambda_{\text {div in }}$ [67].

The filament sizes calculated in this paper are smaller than those reported in previous work $[29,67]$ due to the introduction of 2D cross-correlation for determining the poloidal velocity, and because the trailing wake following the filament is not included when determining the size. The poloidal velocities calculated using the 2D method agree with the force balance equation and are up to $5 \mathrm{x}$ slower than those determined using $1 \mathrm{D}$ cross-correlation, suggesting that the 2D methods are necessary in order to determine the filament sizes accurately. Filament diameters are mainly clustered between 3 and $11 \mathrm{~mm}$ though some filaments are observed to have diameters as large as the distance between the LCFS and the outer wall. Without these improvements, a significant fraction of filaments were found to be much wider than the SOL.

Radial velocities were found mainly clustered in the range of 0.5 to $2 \mathrm{~km} / \mathrm{s}$, but with some filaments having radial velocities as high as $5 \mathrm{~km} / \mathrm{s}$ and as low as $-4 \mathrm{~km} / \mathrm{s}$. The measurements of rapidly inward moving filaments is both surprising and unique as far as we know. It awaits a theoretical explanation, as the theories which do predict inward velocities estimate that the inward velocities should be small compared to the outward ones. The inward moving filaments are only observed in diverted plasmas, with $\mathrm{B}_{\mathrm{t}}>0$ (unfavorable direction for $\mathrm{H}$-mode), and in the region of high poloidal velocity shear within $5 \mathrm{~mm}$ of the LCFS. This measurement is not related to the motion of density holes.

\section{Acknowledgements}

This material is based upon work supported by the U.S. Department of Energy Office of Science, Office of Fusion Energy Sciences under Award Numbers DE-SC0010529 and DE-FG02-97ER54392. The technical contributions of L. Chousal and R. Hernandez are gratefully acknowledged. This work has been carried out within the framework of the EUROfusion Consortium and has received funding from the Euratom research and training programme 2014-2018 under grant agreement No 633053. The views and opinions expressed herein do not necessarily reflect those of the European Commission. This work was supported in part by the Swiss National Science Foundation. B. Lipschultz 
was funded in part by the Wolfson Foundation and UK Royal Society through a Royal Society Wolfson Research Merit Award as well as by the RCUK Energy Programme (EPSRC grant number $\mathrm{EP} / \mathrm{I501045)}$.

\section{$7 \quad$ References}

[1] Hidalgo C Edge turbulence and anomalous transport in fusion plasmas Plasma Phys.

Control. Fusion 37 A53-67 (1995)

https://doi.org/10.1088/07413335/37/11A/004

[2] Horton W Drift waves and transport Rev. Mod. Phys. 71 735-78 (1999)

https://doi.org/10.1103/RevModPhys.71.735

[3] Endler M Turbulent SOL transport in stellarators and tokamaks $J$. Nucl. Mater.

266-269 84-90 (1999)

https://doi.org/10.1016/S0022-

3115(98)00659-X

[4] Carreras B A Progress in anomalous transport research in toroidal magnetic confinement devices IEEE Trans. Plasma Sci. 25 1281-321 (1997)

https://doi.org/10.1109/27.650902

[5] Liewer P C Measurements of microturbulence in tokamaks and comparisons with theories of turbulence and anomalous transport Nucl. Fusion $25543-$ 621 (1985) https://doi.org/10.1088/0029$5515 / 25 / 5 / 004$

[6] Surko C M and Slusher R E Waves and turbulence in a tokamak fusion plasma. Science (80-. ). 221 817-22 (1983) https://doi.org/10.1126/science.221.4613.817

[7] Ritz C P, Lin H, Rhodes T L and Wootton A $\mathrm{J}$ Evidence for confinement improvement by velocity-shear suppression of edge turbulence Phys. Rev. Lett. 65 2543-6 (1990)

https://doi.org/10.1103/PhysRevLett.65.2543

[8] Boedo J A, Terry P W, Gray D, Ivanov R S, Conn R W, Jachmich S and Van Oost G Suppression of temperature fluctuations and energy barrier generation by velocity shear Phys. Rev. Lett. 84 2630-3 (2000) https://doi.org/10.1103/PhysRevLett.84.2630

[9] Tynan G R, Schmitz L, Conn R W, Doerner $\mathrm{R}$ and Lehmer R Steady-state convection and fluctuation-driven particle transport in the $\mathrm{H}$ mode transition Phys. Rev. Lett. 68 3032-5 (1992)

https://doi.org/10.1103/PhysRevLett.68.3032

[10] Biglari H, Diamond P H and Terry P W Influence of sheared poloidal rotation on edge turbulence Phys. Fluids B Plasma Phys. 2 1-4 (1990) https://doi.org/10.1063/1.859529

[11] Team A The H-Mode of ASDEX Nucl. Fusion 29 1959-2040 (1989) https://doi.org/10.1088/0029-5515/29/11/010

[12] Wagner F, Becker G, Behringer K, Campbell D, Eberhagen A, Engelhardt W, Fussmann G, Gehre O, Gernhardt J, Gierke G v., et al Regime of Improved Confinement and High Beta in Neutral-Beam-Heated Divertor Discharges of the ASDEX Tokamak Phys. Rev. Lett. 49 1408-12 (1982)

https://doi.org/10.1103/PhysRevLett.49.1408

[13] LaBombard B, Boivin R L, Greenwald M, Hughes J, Lipschultz B, Mossessian D, Pitcher C S, Terry J L and Zweben S J Particle transport in the scrape-off layer and its relationship to discharge density limit in Alcator C-Mod Phys. Plasmas 8 2107-17 (2001) https://doi.org/10.1063/1.1352596

[14] Carralero D, Birkenmeier G, Müller H W W, Manz P, deMarne P, Müller S H H, Reimold F, Stroth U, Wischmeier M and Wolfrum E An experimental investigation of the high density transition of the scrape-off layer transport in ASDEX Upgrade Nucl. Fusion 54123005 (2014)

https://doi.org/10.1088/00295515/54/12/123005

[15] Carralero D, Manz P, Aho-Mantila L, Birkenmeier G, Brix M, Groth M, Müller H W, Stroth U, Vianello N and Wolfrum E Experimental Validation of a Filament Transport Model in Turbulent Magnetized Plasmas Phys. Rev. Lett. 115215002 (2015) https://doi.org/10.1103/PhysRevLett.115.215 002

[16] Rudakov D L, Boedo J A, Moyer R A, Stangeby P C, Watkins J G, Whyte D G, Zeng L, Brooks N H, Doerner R P, Evans T $\mathrm{E}$, et al Far SOL transport and main wall plasma interaction in DIII-D Nucl. Fusion 45 1589-99 (2005)

https://doi.org/10.1088/0029-5515/45/12/014

[17] Garcia O ., Horacek J, Pitts R ., Nielsen a. ., Fundamenski W, Naulin V and Rasmussen J J Fluctuations and transport in the TCV scrape-off layer Nucl. Fusion 47 667-76 (2007) https://doi.org/10.1088/00295515/47/7/017

[18] Russell D A, Myra J R and D'Ippolito D A Collisionality and magnetic geometry effects on tokamak edge turbulent transport. II. Many-blob turbulence in the two-region model Phys. Plasmas 14102307 (2007) https://doi.org/10.1063/1.2780137

[19] Myra J R, Russell D A and D'Ippolito D A Transport of perpendicular edge momentum 
by drift-interchange turbulence and blobs

Phys. Plasmas 1532304 (2008)

https://doi.org/10.1063/1.2889419

[20] Labit B, Theiler C, Fasoli A, Furno I and Ricci P Blob-induced toroidal momentum transport in simple magnetized plasmas Phys. Plasmas 1832308 (2011) https://doi.org/10.1063/1.3559462

[21] Powers E J Spectral techniques for experimental investigation of plasma diffusion due to polychromatic fluctuations Nucl. Fusion 14 749-52 (1974) https://doi.org/10.1088/0029-5515/14/5/020

[22] Zweben S J Search for coherent structure within tokamak plasma turbulence Phys. Fluids 28974 (1985) https://doi.org/10.1063/1.865069

[23] Wootton A J, Carreras B A, Matsumoto H, McGuire K, Peebles W A, Ritz C P, Terry P $\mathrm{W}$ and Zweben S J Fluctuations and anomalous transport in tokamaks Phys. Fluids B Plasma Phys. 2 2879-903 (1990) https://doi.org/10.1063/1.859358

[24] Goodall D H J High speed cine film studies of plasma behaviour and plasma surface interactions in tokamaks $J$. Nucl. Mater. 111112 11-22 (1982) https://doi.org/10.1016/0022-3115(82)90174$\mathrm{X}$

[25] Filippas A V., Bengston R D, Li G -X., Meier M, Ritz C P and Powers E J Conditional analysis of floating potential fluctuations at the edge of the Texas Experimental Tokamak Upgrade (TEXT-U) Phys. Plasmas 2 839-45 (1995) https://doi.org/10.1063/1.871435

[26] Zweben S J, Stotler D P, Terry J L, Labombard B, Greenwald M, Muterspaugh M, Pitcher C S, Hallatschek K, Maqueda R J, Rogers B, et al Edge turbulence imaging in the Alcator C-Mod tokamak Physics of Plasmas vol 9pp 1981-9 (2002) https://doi.org/10.1063/1.1445179

[27] Krasheninnikov S I On scrape off layer plasma transport Phys. Lett. Sect. A Gen. At. Solid State Phys. 283 368-70 (2001) https://doi.org/10.1016/S03759601(01)00252-3

[28] D'Ippolito D A, Myra J R and Krasheninnikov S I Cross-field blob transport in tokamak scrape-off-layer plasmas Phys. Plasmas 9222 (2002) https://doi.org/10.1063/1.1426394

[29] Boedo J A, Rudakov D, Moyer R, Krasheninnikov S, Whyte D, McKee G, Tynan G, Schaffer M, Stangeby P, West P, et al Transport by intermittent convection in the boundary of the DIII-D tokamak Phys.

Plasmas 8 4826-33 (2001)

https://doi.org/10.1063/1.1406940

[30] Rudakov D L, Boedo J A, Moyer R A, Krasheninnikov S, Leonard A W, Mahdavi M A, McKee G R, Porter G D, Stangeby P C, Watkins J G, et al Fluctuation-driven transport in the DIII-D boundary Plasma Phys. Control. Fusion 44 717-31 (2002) https://doi.org/10.1088/0741-3335/44/6/308

[31] Boedo J A, Rudakov D L, Moyer R A, McKee G R, Colchin R J, Schaffer M J, Stangeby P G, West W P, Allen S L, Evans T $\mathrm{E}$, et al Transport by intermittency in the boundary of the DIII-D tokamak Phys. Plasmas 10 1670-7 (2003) https://doi.org/10.1063/1.1563259

[32] Myra J R, Russell D A and D'Ippolito D A Collisionality and magnetic geometry effects on tokamak edge turbulent transport. I. A two-region model with application to blobs Phys. Plasmas 13112502 (2006) https://doi.org/10.1063/1.2780137

[33] Myra J R, D’Ippolito D a., Xu X Q and Cohen R H Resistive modes in the edge and scrape-off layer of diverted tokamaks Phys. Plasmas 74622 (2000) https://doi.org/10.1063/1.1314623

[34] D'Ippolito D A and Myra J R Blob stability and transport in the scrape-off-layer Phys.

Plasmas 10 4029-39 (2003) https://doi.org/10.1063/1.1606447

[35] Russell D A, D’Ippolito D A, Myra J R, Nevins $\mathrm{W} M$ and $\mathrm{Xu} X \mathrm{Q}$ Blob Dynamics in 3D BOUT Simulations of Tokamak Edge Turbulence Phys. Rev. Lett. 93265001 (2004) https://doi.org/10.1103/PhysRevLett.93.2650 01

[36] Pigarov A Y, Krasheninnikov S I, Rognlien T D, Schaffer M J and West W P Tokamak edge plasma simulation including anomalous cross-field convective transport Phys.

Plasmas 9 1287-99 (2002) https://doi.org/10.1063/1.1459059

[37] Krasheninnikov S I, D'Ippolito D A and Myra J R Recent theoretical progress in understanding coherent structures in edge and SOL turbulence J. Plasma Phys. 74 679-717 (2008) https://doi.org/10.1017/S0022377807006940

[38] Myra J R and D'Ippolito D A Edge instability regimes with applications to blob transport and the quasicoherent mode Phys. Plasmas 12 1-10 (2005) https://doi.org/10.1063/1.2048847

[39] Aydemir A Y Convective transport in the 
scrape-off layer of tokamaks Phys. Plasmas 1262503 (2005) https://doi.org/10.1063/1.1927539

[40] Yu G Q and Krasheninnikov S I Dynamics of blobs in scrape-off-layer/shadow regions of tokamaks and linear devices Phys.

Plasmas 10 4413-8 (2003) https://doi.org/10.1063/1.1616937

[41] D'Ippolito D A and Myra J R Thermal transport catastrophe and the tokamak edge density limit Phys. Plasmas 1362503 (2006) https://doi.org/10.1063/1.2206168

[42] Krasheninnikov S I and Smolyakov A I On neutral wind and blob motion in linear devices Phys. Plasmas 10 3020-1 (2003) https://doi.org/10.1063/1.1579692

[43] Daughton W, Catto P J, Coppi B and Krasheninnikov S I Interchange instabilities in a partially ionized plasma Phys. Plasmas $\mathbf{5}$ 2217 (1998) https://doi.org/10.1063/1.872895

[44] Katz N, Egedal J, Fox W, Le A and Porkolab M Experiments on the Propagation of Plasma Filaments Phys. Rev. Lett. 10115003 (2008) https://doi.org/10.1103/PhysRevLett.101.015 003

[45] Militello F, Dudson B, Easy L, Kirk A and Naylor P On the interaction of scrape off layer filaments Plasma Phys. Control. Fusion 59125013 (2017) https://doi.org/10.1088/1361-6587/aa9252

[46] Myra J R, D’Ippolito D A, Stotler D P, Zweben S J, Leblanc B P, Menard J E, Maqueda R J, Boedo J, D 'ippolito D A, Stotler D P, et al Blob birth and transport in the tokamak edge plasma: Analysis of imaging data Phys. Plasmas 1392509 (2006) https://doi.org/10.1063/1.2355668

[47] Theiler C, Furno I, Fasoli A, Ricci P, Labit B and Iraji D Blob motion and control in simple magnetized plasmas Phys. Plasmas 1855901 (2011) https://doi.org/10.1063/1.3562944

[48] Myra J R, D’Ippolito D A, Krasheninnikov S $\mathrm{I}$ and $\mathrm{Yu} \mathrm{G} \mathrm{Q}$ Convective transport in the scrape-off-layer by nonthermalized spinning blobs Phys. Plasmas 11 4267-74 (2004) https://doi.org/10.1063/1.1774168

[49] D'Ippolito D A, Myra J R, Russell D A and Yu G Q Rotational stability of plasma blobs Phys. Plasmas 11 4603-9 (2004)

https://doi.org/10.1063/1.1785791

[50] Angus J R, Umansky M V. and Krasheninnikov S I Effect of Drift Waves on Plasma Blob Dynamics Phys. Rev. Lett. 108 215002 (2012) https://doi.org/10.1103/PhysRevLett.108.215 002

[51] Ryutov D D The dynamics of an isolated plasma filament at the edge of a toroidal device Phys. Plasmas 13122307 (2006) https://doi.org/10.1063/1.2403092

[52] Walkden N R, Dudson B D and Fishpool G Characterization of 3D filament dynamics in a MAST SOL flux tube geometry Plasma Phys. Control. Fusion 55105005 (2013) https://doi.org/10.1088/07413335/55/10/105005

[53] Easy L, Militello F, Omotani J, Dudson B, Havlíčková E, Tamain P, Naulin V and Nielsen A H Three dimensional simulations of plasma filaments in the scrape off layer: A comparison with models of reduced dimensionality Phys. Plasmas 21122515 (2014) https://doi.org/10.1063/1.4904207

[54] Mosetto A, Halpern F D, Jolliet S, Loizu J and Ricci P Turbulent regimes in the tokamak scrape-off layer Phys. Plasmas 2092308 (2013) https://doi.org/10.1063/1.4821597

[55] Bisai N, Singh R and Kaw P K Scrape-off layer tokamak plasma turbulence $P$ hys. Plasmas 1952509 (2012)

https://doi.org/10.1063/1.4718714

[56] Manz P, Birkenmeier G, Carralero D, Fuchert G, Müller H W, Müller S H, Scott B D, Stroth U, Ribeiro T T and Wolfrum E The influence of finite ion temperature on plasma blob dynamics Plasma Phys. Control. Fusion 5714012 (2015)

https://doi.org/10.1088/07413335/57/1/014012

[57] Russell D A, D'Ippolito D A, Myra J R, Canik J M, Gray T K and Zweben S J Modeling the effect of lithium-induced pedestal profiles on scrape-off-layer turbulence and the heat flux width Phys. Plasmas 2292311 (2015) https://doi.org/10.1063/1.4930285

[58] Solano E R and Hazeltine R D Magnetic phase transitions in plasmas and transport barriers Nucl. Fusion 52114017 (2012) https://doi.org/10.1088/00295515/52/11/114017

[59] Omotani J T, Militello F, Easy L and Walkden N R The effects of shape and amplitude on the velocity of scrape-off layer filaments Plasma Phys. Control. Fusion 58 14030 (2016) https://doi.org/10.1088/07413335/58/1/014030

[60] Madsen J, Garcia O E, Stærk Larsen J, Naulin V, Nielsen A H and Rasmussen J J The influence of finite Larmor radius effects on the radial interchange motions of plasma filaments Phys. Plasmas 18112504 (2011) https://doi.org/10.1063/1.3658033

[61] Wiesenberger M, Madsen J and Kendl A 
Radial convection of finite ion temperature, high amplitude plasma blobs Phys. Plasmas 2192301 (2014) https://doi.org/10.1063/1.4894220

[62] Bodi K, Krasheninnikov S I and Smolyakov A I Blob dynamics in an inhomogeneous plasma Phys. Plasmas 15102304 (2008) https://doi.org/10.1063/1.2993211

[63] Kendl A Inertial blob-hole symmetry breaking in magnetised plasma filaments Plasma Phys. Control. Fusion 5745012 (2015) https://doi.org/10.1088/07413335/57/4/045012

[64] Garcia O E, Kube R, Theodorsen A and Pécseli H L Stochastic modelling of intermittent fluctuations in the scrape-off layer: Correlations, distributions, level crossings, and moment estimation Phys. Plasmas 2352308 (2016) https://doi.org/10.1063/1.4951016

[65] Graves J P, Horacek J, Pitts R A and Hopcraft K I Self-similar density turbulence in the TCV tokamak scrape-off layer Plasma Phys. Control. Fusion 47 L1-9 (2005) https://doi.org/10.1088/0741-3335/47/3/L01

[66] Garcia O E, Pitts R A, Horacek J, Nielsen A H, Fundamenski W, Graves J P, Naulin V and Rasmussen J J Turbulent transport in the TCV SOL J. Nucl. Mater. 363-365 575-80 (2007) https://doi.org/10.1016/j.jnucmat.2006.12.06 3

[67] Vianello N, Tsui C, Theiler C, Allan S, Boedo J, Labit B, Reimerdes H, Verhaegh K, Vijvers W A J, Walkden N, et al

Modification of SOL profiles and fluctuations with line-average density and divertor flux expansion in TCV Nucl. Fusion 57116014 (2017) https://doi.org/10.1088/17414326/aa7db3

[68] Garcia O E, Horacek J, Pitts R A, Nielsen A H, Fundamenski W, Graves J P, Naulin V and Rasmussen J J Interchange turbulence in the TCV scrape-off layer Plasma Phys.

Control. Fusion 48 L1-10 (2006) https://doi.org/10.1088/0741-3335/48/1/L01

[69] Garcia O E, Pitts R A, Horacek J, Madsen J, Naulin V, Nielsen A H and Rasmussen J J Collisionality dependent transport in $T C V$ SOL plasmas vol 49(IOP Publishing) (2007) https://doi.org/10.1088/0741$3335 / 49 / 12 \mathrm{~B} / \mathrm{S} 03$

[70] Theodorsen A, Garcia O E, Horacek J, Kube R and Pitts R A Scrape-off layer turbulence in TCV: Evidence in support of stochastic modelling Plasma Phys. Control. Fusion 58 44006 (2016) https://doi.org/10.1088/0741-
$3335 / 58 / 4 / 044006$

[71] Garcia O E, Horacek J and Pitts R A Intermittent fluctuations in the TCV scrapeoff layer Nucl. Fusion 5562002 (2015) https://doi.org/10.1088/00295515/55/6/062002

[72] Theiler C, Furno I, Ricci P, Fasoli A, Labit B, Müller S H and Plyushchev G Cross-field motion of plasma blobs in an open magnetic field line configuration Phys. Rev. Lett. 103 65001 (2009)

https://doi.org/10.1103/PhysRevLett.103.065 001

[73] Zweben S J, Myra J R, Davis W M, D'Ippolito D A, Gray T K, Kaye S M, Leblanc B P, Maqueda R J, Russell D A and Stotler D P Blob structure and motion in the edge and SOL of NSTX Plasma Phys.

Control. Fusion 5844007 (2016)

https://doi.org/10.1088/07413335/58/4/044007

[74] Carralero D, Müller H W, Groth M, Komm M, Adamek J, Birkenmeier G, Brix M, Janky F, Hacek P, Marsen S, et al Implications of high density operation on SOL transport: A multimachine investigation $J$. Nucl. Mater. 463 123-7 (2015) https://doi.org/10.1016/j.jnucmat.2014.10.01 9

[75] Krasheninnikov S, Ryutov D and Yu G Large plasma pressure perturbations and radial convective transport in a tokamak $J$. Plasma Fusion Res. 6 (2004)

[76] Myra J R, D'Ippolito D A, Xu X Q and Cohen R H Resistive X-point modes in tokamak boundary plasmas Phys. Plasmas 7 2290 (2000) https://doi.org/10.1063/1.874125

[77] Garcia O E, Bian N H, Naulin V, Nielsen A $\mathrm{H}$ and Rasmussen $\mathrm{J} J$ Mechanism and scaling for convection of isolated structures in nonuniformly magnetized plasmas Phys. Plasmas 1290701 (2005) https://doi.org/10.1063/1.2044487

[78] Garcia O E, Bian N H and Fundamenski W Radial interchange motions of plasma filaments Phys. Plasmas 1382309 (2006) https://doi.org/10.1063/1.2336422

[79] Furno I, Theiler C, Lançon D, Fasoli A, Iraji D, Ricci P, Spolaore M and Vianello N Blob current structures in TORPEX plasmas: experimental measurements and numerical simulations Plasma Phys. Control. Fusion 53 124016 (2011) https://doi.org/10.1088/07413335/53/12/124016

[80] Zweben S J, Davis W M, Kaye S M, Myra J R, Bell R E, LeBlanc B P, Maqueda R J, Munsat T, Sabbagh S A, Sechrest Y, et al 
Edge and SOL turbulence and blob variations over a large database in NSTX Nucl. Fusion 5593035 (2015)

https://doi.org/10.1088/0029-

5515/55/9/093035

[81] Theodorsen A and Garcia O E Level crossings, excess times, and transient plasma-wall interactions in fusion plasmas Phys. Plasmas 2340702 (2016) https://doi.org/10.1063/1.4947235

[82] Nespoli F, Labit B, Furno I, Horacek J, Tsui C K, Boedo J A, Maurizio R, Reimerdes H, Theiler C, Ricci P, et al Understanding and suppressing the near scrape-off layer heat flux feature in inboard-limited plasmas in TCV Nucl. Fusion 57126029 (2017) https://doi.org/10.1088/1741-4326/aa84e0

[83] Tsui C K, Boedo J A, Halpern F D, Loizu J, Nespoli F, Horacek J, Labit B, Morales J, Reimerdes H, Ricci P, et al Poloidal asymmetry in the narrow heat flux feature in the TCV scrape-off layer Phys. Plasmas 24 62508 (2017)

https://doi.org/10.1063/1.4985075

[84] Verhaegh K, Lipschultz B, Duval B P, Harrison J R, Reimerdes H, Theiler C, Labit B, Maurizio R, Marini C, Nespoli F, et al Spectroscopic investigations of divertor detachment in TCV Nucl. Mater. Energy $01-$ 6 (2016) https://doi.org/10.1016/j.nme.2017.01.004

[85] Harrison J R, Vijvers W A J, Theiler C, Duval B P, Elmore S, Labit B, Lipschultz B, van Limpt S H M, Lisgo S W, Tsui C K, et al Detachment evolution on the TCV tokamak Nucl. Mater. Energy 12 1071-6 (2017) https://doi.org/10.1016/j.nme.2016.10.020

[86] Theiler C, Lipschultz B, Harrison J, Labit B, Reimerdes H, Tsui C K, Vijvers W A J A J, Boedo J A, Duval B P P, Elmore S, et al Results from recent detachment experiments in alternative divertor configurations on TCV Nucl. Fusion 5772008 (2017) https://doi.org/10.1088/1741-4326/aa5fb7

[87] Boedo J A, Crocker N, Chousal L, Hernandez R, Chalfant J, Kugel H, Roney P and Wertenbaker J Fast scanning probe for the NSTX spherical tokamak Rev. Sci. Instrum. 80123506 (2009) https://doi.org/10.1063/1.3266065

[88] Tsui C K, Boedo J A, Stangeby P C and Team T Accounting for Debye sheath expansion for proud Langmuir probes in magnetic confinement fusion plasmas Rev. Sci. Instrum. 8913505 (2018) https://doi.org/10.1063/1.4995353

[89] Lister J B, Hofmann F, Moret J-M,
Bühlmann F, Dutch M J, Fasel D, Favre A, Isoz P-F, Marletaz B, Marmillod P, et al The control of TCV plasmas Fusion Sci. Technol. 32 321-73 (1997)

[90] Garcia O E, Naulin V, Nielsen A H and Rasmussen J J Turbulence simulations of blob formation and radial propagation in toroidally magnetized plasmas Phys. Scr. T122 89-103 (2006) https://doi.org/10.1088/00318949/2006/T122/013

[91] McKee G R, Petty C C, Waltz R E, Fenzi C, Fonck R J, Kinsey J E, Luce T C, Burrell K H, Baker D R, Doyle E J, et al Nondimensional scaling of turbulence characteristics and turbulent diffusivity $\mathrm{Nucl}$. Fusion 41 1235-42 (2001) https://doi.org/10.1088/0029-5515/41/9/312

[92] Ritz C P, Powers E J, Rhodes T L, Bengtson R D, Gentle K W, Lin H, Phillips P E, Wootton A J, Brower D L, Luhmann N C, et al Advanced plasma fluctuation analysis techniques and their impact on fusion research (invited) Rev. Sci. Instrum. 59 173944 (1988) https://doi.org/10.1063/1.1140098

[93] Brotankova J, Stockel J, Horacek J, Seidl J, Duran I, Hron M and Oost G Measurement of sheared flows in the edge plasma of the CASTOR tokamak Plasma Phys. Reports 35 980 (2009)

https://doi.org/10.1134/S1063780X09110087

[94] Fedorczak N, Manz P, Thakur S C, Xu M, Tynan G R, Xu G S and Liu S C On physical interpretation of two dimensional timecorrelations regarding time delay velocities and eddy shaping Phys. Plasmas 19122302 (2012) https://doi.org/10.1063/1.4769849

[95] Groebner R J, Burrell K H and Seraydarian R P Role of edge electric field and poloidal rotation in the L-H transition Phys. Rev. Lett. 64 3015-8 (1990) https://doi.org/10.1103/PhysRevLett.64.3015

[96] LaBombard B An interpretation of fluctuation induced transport derived from electrostatic probe measurements Phys. Plasmas 91300 (2002) https://doi.org/10.1063/1.1459060

[97] Boedo J A, Myra J R, Zweben S, Maingi R, Maqueda R J, Soukhanovskii V A, Ahn J W, Canik J, Crocker N, D'Ippolito D A, et al Edge transport studies in the edge and scrapeoff layer of the National Spherical Torus Experiment with Langmuir probes Phys. Plasmas 21 (2014) https://doi.org/10.1063/1.4873390

[98] Rudakov D L, Boedo J A, Moyer R A, Lehmer R D, Gunner G and Watkins J G Fast 
electron temperature diagnostic based on Langmuir probe current harmonic detection on DIII-D Rev. Sci. Instrum. 72 453-6 (2001) https://doi.org/10.1063/1.1310577

[99] D'Ippolito D A, Myra J R and Zweben S J Convective transport by intermittent blobfilaments: Comparison of theory and experiment Phys. Plasmas 1860501 (2011) https://doi.org/10.1063/1.3594609

[100] Russell D A, Myra J R, D'Ippolito D A, Munsat T L, Sechrest Y, Maqueda R J, Stotler D P and Zweben S J Comparison of scrape-off layer turbulence simulations with experiments using a synthetic gas puff imaging diagnostic Phys. Plasmas 18 (2011) https://doi.org/10.1063/1.3553024

[101] Myra J R, Davis W M, D'Ippolito D A, LaBombard B, Russell D A, Terry J L and Zweben S J Edge sheared flows and the dynamics of blob-filaments Nucl. Fusion 53 73013 (2013) https://doi.org/10.1088/00295515/53/7/073013

[102] Easy L, Militello F, Omotani J, Walkden N $\mathrm{R}$ and Dudson B Investigation of the effect of resistivity on scrape off layer filaments using three-dimensional simulations Phys. Plasmas 2312512 (2016)

https://doi.org/10.1063/1.4940330 